\title{
Termodinâmica aplicada à teoria de jogos: estudo sobre risco associado à cooperação no jogo do Bem Público
}

Dissertação apresentada à Faculdade de Filosofia, Ciências e Letras de Ribeirão Preto da Universidade de São Paulo como parte das exigências para a obtenção do título de Mestre em Ciências.

Área de Concentração:

Física Aplicada à Medicina e Biologia.

Orientador:

Alexandre Souto Martinez.

Versão corrigida

Versão original disponível na FFCLRP-USP

Ribeirão Preto 
Autorizo a reprodução e divulgação total ou parcial deste trabalho, por qualquer meio convencional ou eletrônico, para fins de estudo e pesquisa, desde que citada a fonte.

\section{FICHA CATALOGRÁFICA}

dos Santos, Guilherme Contesini

Termodinâmica aplicada à teoria de jogos: estudo sobre risco associado à cooperação no jogo do Bem Público / Guilherme Contesini dos Santos; orientador: Alexandre Souto Martinez. - - Ribeirão Preto, 2019.

$36 \mathrm{f}$. : il.

Dissertação (Mestrado) - - Faculdade de Filosofia, Ciências e Letras de Ribeirão Preto, Universidade de São Paulo, 2019.

Inclui Bibliografia.

1. Teoria de Jogos 2. Termodinâmica 3. Mecanica Estatística 4. Jogos Potencionais Evolucionarios. 
Nome: Dos SAntos, Guilherme Contesini

Título: Termodinâmica aplicada à teoria de jogos: estudo sobre risco associado à cooperação no jogo do Bem Público

Dissertação apresentada á Faculdade de Filosofia, Ciências e Letras de Ribeirão Preto da Universidade de São Paulo como parte das exigências para a obtenção do título de Mestre em Ciências.

Aprovado em:

\section{Banca Examinadora}

$\operatorname{Prof}(\mathrm{a}) \cdot \operatorname{Dr}(\mathrm{a})$.

Instituição:

Julgamento:

Assinatura:

$\operatorname{Prof}(\mathrm{a}) \cdot \operatorname{Dr}(\mathrm{a})$.

Instituição:

Julgamento:

Assinatura:

Prof(a). Dr(a). :

Instituição:

Julgamento:

Assinatura: 


\section{Agradecimentos}

Ao meu pai, a minha mãe e a minha irmã por toda ajuda e o apoio nos mometos mais difíceis.

Ao meu orientador Prof. Dr. Alexandre S. Martinez pela orientação, críticas e ensinamentos nestes últimos dois anos.

Aos meus professores Dr. Luiz C. Mostaço-Guidolin e Dr. Aquino L. de Espíndola pela ajuda, indicação e orientação,

Aos colegas de laboratório Gilberto, José Renato, Alexandre DiMartini Cristiano, Fernando e Claudio pelo apoio, ensinamentos, conversas doidas, risadas e pelo café,

Ao Prof. Dr. Marcelo Mulato pelos ensinamentos e críticas durante o estágio do Programa de Aperfeiçoamento de Ensino.

Aos grandes amigos que fiz ao longo do meu mestrado,

Aos professores da FAMB que contribuiram para minha formação,

À Coordenação de Aperfeiçoamento de Pessoal de Nível Superior (CAPES) pelo apoio financeiro à minha pesquisa e ao departamento - Código de Financiamento 001.

Ao Instituto Nacional de Ciência e Tecnologia de Sistemas Complexos INCT-SC pelo apoio financeiro dado ao grupo de pesquisa, Laboratório de Modelagem de Sistemas Complexos. 


\section{Resumo}

CONTESINI, G. S. Termodinâmica aplicada à teoria de jogos: estudo sobre risco associado à cooperação no jogo do Bem Público. 2019. 36 f. Dissertação (Mestrado - Programa de Pós-Graduação em Física Aplicada à Medicina e Biologia) - Faculdade de Filosofia, Ciências e Letras de Ribeirão Preto, Universidade de São Paulo, Ribeirão Preto, 2019.

A emergência dos dilemas de cooperação é um dos fenômenos que ainda não compreendemos completamente. Observamos muitos exemplos dos dilemas de cooperação na natureza. Os jogos potenciais são uma alternativa viável para estudar o comportamento realista dos jogadores. Este formalismo estabelece uma ponte entre a Teoria de Jogos e conceitos da Termodinâmica. Inspirado nos estudos recentes deste formalismo, estudamos o jogo do Bem Público utilizando ferramentas da Mecânica Estatística. Introduzimos uma notação de operadores, descrevendo o ganho dos jogadores em termos equações de autovalores e autovetores. Estudamos o jogo do Bem Público, descrevendo-o através de uma "Hamiltoniana". A análise entre o operador ganho individual e o ganho coletivo, no cenário mais simples do jogo, revela que o dilema emerge quando escolhemos apenas um operador para descrever o jogo. Definimos o operador "risco de cooperação" e identificamos que ele é necessário para descrever o Equilíbrio de Nash correto. Estudamos também o mecanismo de punição no jogo do Bem Público. Observamos o surgimento de uma transiçãos de fase, que segrega o regime cooperativos do regime competitivos.

Palavras-chave: 1. Teoria de Jogos 2. Termodinâmica 3. Mecanica Estatística 4. Jogos Potencionais Evolucionarios. 


\section{Abstract}

CONTESINI, G. S. Thermodinamics applied to game theory: study of the risk associated with cooperation in the Public Goods Games. 2019. 36 f. Dissertation (M.Sc. Postgraduate program in Physics Applied to Medicine and Biology) - Faculty of Philosophy, Sciences and Letters, University of São Paulo, Ribeirão Preto, 2019.

The emergence of cooperation dilemmas is a phenomenon that we still do not fully understand. We observe many examples of these dilemmas in nature. Potential games are a feasible alternative to study the realistic behavior in players. This formalism establishes a connection between the Game Theory and concepts of Thermodynamics. Inspired by the recent studies of this formalism, we have studied the Public Goods game using Statistical Mechanics tools. We introduce an operatorial notation, describing the players's payoff in terms of eigenvalues and eigenvectors equations. We study the Public Good Game, describing it through a "Hamiltonian". The analysis between the individual and the collective payoff operators, in the simplest scenario of the game, reveals that the dilemma emerges when we choose which operator describe the game. We created the "risk of cooperation" operator and identified that it is necessary to describe the correct Nash equilibrium. We also study the mechanism of punishment in the game of Public Goods Game. We observed the emergence of phase transitions, and that these transitions divide the cooperative regime from desertion regime.

Key-words: 1. Game Theory 2. Thermodinamics 3. Statistical Physics 4. Evolutionary Potential Games 


\section{Lista de Figuras}

5.1 Arranjo dos jogadores em um linha completa fornece a condição de contorno periódica para o sistema ${ }^{1}$. . . . . . . . . . . . . 40

5.2 Observamos o crescimento monotônico da estratégia média por jogador, indicando que, para os valores $(\beta \gg 1)$ os jogadores tendem a cooperar. Este resultado não corrobora com o Equilíbrio de Nash previsto pela teoria para o $J B P$. Isto sugere que um fator intrínseco que afeta a decisão dos jogadores e corrige a dinâmica do jogo ${ }^{2}$. Em cinza é expresso a região de incerteza. . . . . . . . . . . . . . . . . . 41

5.3 Estratégia média esperada quando o risco de cooperação é considerado $^{3}$. Novamente observamos um comportamento monotonicamente, porém não exclusivamente crescente. A incerteza (SE) é representada pela região cinza. . . . . . . . . . . . . . . . . . . . . . 44

5.4 Representação esquemática do $J B P$ com punição para um grafo completo. . . . . . . . . . . . . . . . . . 45

5.5 O gráfico mostra o comportamento da estratégia média por jogador com a redução do ruído. Percebe-se que a punição promove a cooperação, inclusive no cenário de indecisão $c / b=0.5^{4} \ldots \ldots$. . . . . . 47 
5.6 O gráfico mostra o comportamento da estratégia média por jogador para os valores $c / b \sim 0.65$. O gráfico mostra que a punição muda abruptamente o comportamento dos jogadores. Para valores $c / b>$ 0.75 e $\beta>\beta^{*} \sim 1.3$ a punição passa a promover a deserção ${ }^{5} . . .48$

5.7 A análise do espaço de parâmetros custo-ganho com punição máxima $\gamma=1$ no limiar de um mudança de regimes cooperação-deserção, revela um sinal de uma possível transição de fase crítica. A condição de transição de fase crítica é observada após uma pequena redução na relação custo-ganho com punção máxima $\gamma=1^{6}$. . . . . . . . . . 49

5.8 O gráfico mostra o comportamento da variância da densidade de estratégias com o aumento de $\beta$ na escala logarítmica. Observamos o surgimento da transição de fase para a condição vista anteriormente $(c / b=0.665)$ com punição máxima $(\gamma=1)$ com o aumento de $\beta$. A variância revela que a divergência ocorre para valores próximos de

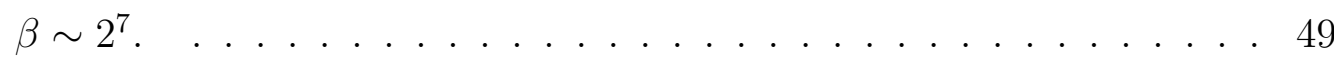

5.9 Comportamento da variância na escala log-log. O gráfico mostra uma transição de fase tipo lambda que decai com a lei $\left|\beta-\beta_{0}\right|^{-\omega_{1}} e^{-\omega_{2} \beta 8} . \quad 50$ 


\section{Sumário}

Lista de Figuras $\quad$ xi

1 Introdução 1

1.1 A evolução da Teoria de Jogos e suas aplicações . . . . . . . . . . . . 2

1.2 Dissertação . . . . . . . . . . . . . . . . . . . 5

1.3 Resultados e organização da Dissertação . . . . . . . . . . . . . . . . 6

$\begin{array}{lll}2 & \text { A Teoria de Jogos } & 9\end{array}$

2.1 Fundamentos da Teoria de Jogos . . . . . . . . . . . . . . . . . . . . 9

2.2 Estratégias Dominantes e o Equilíbrio de Nash . . . . . . . . . . . 15

2.3 O Jogo do Bem Público . . . . . . . . . . . . . . . . 16

3 A Teoria de Jogos Evolucionários $\quad 19$

3.1 Teoria de Jogos Evolucionários . . . . . . . . . . . . . . . . 20

3.2 Estratégias Evolutivas _. . . . . . . . . . . . . . . . . . 22

4 Jogos Potenciais Evolucionários $\quad 27$

4.1 Jogos potenciais . . . . . . . . . . . . . . . . 27

4.2 Conexão entre estratégias estocásticas e a termodinâmica . . . . . . 29

5 Resultados $\quad 35$

5.1 Notação algébrica para os jogos potenciais evolutivos . . . . . . . . . 35 
5.2 Modelo de spins para o Jogo do Bem Público . . . . . . . . . . . . . 37

5.2.1 Jogo do Bem Público com punição aos desertores. . . . . . . . 44

6 Conclusão

Referências Bibliográficas 


\title{
Introdução
}

\author{
Não existe conversa mais tediosa do que aquela na qual todas as \\ partes concordam entre si. \\ - Michel de Montaigne
}

O surgimento de dilemas de cooperação é um fenômeno que instiga o nosso conhecimento sobre a natureza. Um dilema de cooperação é o surgimento de um comportamento inesperado entre indivíduos, ou seja, é a traição entre indivíduos em situações favoráveis à cooperação, ou vice-versa. Existem muitos exemplos destes dilemas na natureza, os quais ainda não compreendemos. Situações como, a formação de cardumes, alcateias de leões, colaboração entre golfinhos e pescadores ou surgimento de organismos multicelulares a partir de unicelulares são exemplos destes dilemas [1-7]. Desde do século $X I X$, estes dilemas intrigam diferentes áreas do conhecimento como Filosofia, Ciências Políticas, Economia, Biologia e Sociologia, como descrito por Alfred R. Wallace [8]:

À primeira vista, parece que tais comportamentos como os de justiça abstrata e benevolência nunca poderiam ter sido adquiridos, porque eles são incompatíveis com a lei do mais forte, que é a essência da seleção natural. Mas isto é, penso eu, uma visão errônea, porque devemos olhar não para indivíduos, mas para as sociedades; e justiça e benevolência, exercidas com respeito aos membros da mesma tribo, certamente tenderiam a fortalecer essa tribo, e darem-lhe uma superioridade sobre a outra, cuja a regra a do mais forte prevaleceu, onde consequentemente 
os fracos e doentes foram deixados a perecer, e os mais fortes destruíram impiedosamente aqueles que eram mais fracos. ${ }^{1}$

(Alfred R. Wallace - Contribuições à teoria da seleção natural - Capítulo 'Os limites da Seleção Natural aplicados ao homen' - página 351)

O pensamento que emerge desse argumento é que a seleção natural não seleciona apenas as "aptidões" dos indivíduos, mas também a "aptidão" do grupo, que através de um mecanismo interno regula o comportamento dos indivíduos. O dilema de cooperação emerge quando os indivíduos egoístas exploram um comportamento cooperativo, prejudicando o grupo. Verificamos isso na natureza, ao observarmos situações onde a formação de grupos sociais parecem violar a lei da seleção natural [9-11]. Em vários sistemas biológicos, indivíduos fracos ou doentes são favorecidos pelo comportamento coletivo, permitindo que sobrevivam e reproduzam. A Seleção Natural, base da teoria da evolução de Charles Darwin e Alfred Wallace [12], expõe os reais conflitos nos dilemas de cooperação. O estudo do dilema de cooperação nos revela perguntas mais abrangentes sobre o comportamento de indivíduos e grupos. Por que observamos, em um mesmo cenário, a cooperação e a traição entre indivíduos? Por que traímos em situações onde a cooperação é a mais vantajosa e vice-versa? Quais os fatores influenciam a cooperação (traição)? Essas perguntas são cruciais para a solução dos dilemas de cooperação. A teoria de Darwin e Wallace apresenta essas perguntas, porém demonstra limitações ao tornar precisas as relações coletivas.

\subsection{A evolução da Teoria de Jogos e suas aplicações}

Criada inicialmente para cenários econômicos [13], a teoria dos jogos emerge naturalmente na biologia quando descrevemos situações de conflito que apresentam os dilemas de cooperação. Um jogo da Teoria de Jogos é uma descrição abstrata de

\footnotetext{
${ }^{1}$ Tradução feita pelo autor. [8]
} 
um processo de tomada de decisão em analogia a uma situação real. A descrição concisa dos dilemas, por meio dos jogos, possibilita uma análise quantitativa do comportamento dos indivíduos, grupos, e consequentemente dos fatores que influenciam a cooperação, ou traição $[13,14]$.

Apesar de seu sucesso, a Teoria de Jogos é considerada uma teoria relativamente nova. O formalismo dos jogos foi introduzido pela primeira vez em 1928 por von Neumman e Oskar Morgenstern para modelar processos de decisão na economia [13]. Os primeiros jogos e teoremas foram formulados para jogos estritamente competitivos, chamados de jogos de soma nula, nos quais o ganho de um jogador é o prejuízo do outro. No início, a teoria de jogos não se preocupava com os dilemas de cooperação: o seu o principal objetivo era identificar as estratégias que maximizavam o lucro e reduziam as perdas [15]. Jogos como o "par ou impar", o "jogo do ultimato"2 e o Poker são exemplos dessa classe de jogos. A aplicação de jogos de soma nula está limitada ao vínculo, se um jogador ganha o outro necessariamente perde. Esses jogos eram usados, por exemplo, em cenários econômicos e políticos, em que havia sempre um vencedor e um perdedor. Exemplos destes jogos aparecem, na economia, em mercados de opções e em contrato futuros, e na politica, nas "corridas" presidenciais e votações de projetos leis [16].

Com o surgimento de novos jogos, como "o dilema do prisioneiro", "o problema da barganha", "o jogo bem público" e "a batalha dos sexos" [17-19], surgia a possibilidade de resultados onde a cooperação entre os jogadores era mais propícia, ou seja, surgia os jogos de soma não nula. A teoria dos jogos passou, portanto, a descrever com maior naturalidade os dilemas de cooperação. Com os jogos de soma não nula surge a necessidade de um conceito de estabilidade, uma solução que sugere qual comportamento é mais aguardado entre os jogadores. Esse novo conceito surge

\footnotetext{
2No "jogo do ultimato", dois jogadores devem decidir como dividir uma determinada quantia de dinheiro. Neste jogo, o primeiro jogador faz uma proposta, enquanto o segundo jogador decidi se aceita ou não. Caso a divisão não for aceita ambos não recebem nada.
} 
com os estudos de John F. Nash, que além de revolucionarem a teoria até o momento, popularizaram e ampliaram seu escopo e aplicações de jogos para diferentes áreas $[20-22]$.

Uma área que foi beneficiada com a desenvolvimento da Teoria de Jogos foi a Biologia. Não levou muito tempo até que biólogos notassem a semelhança entre os modelos propostos pela teoria de jogos e as interações entre organismos e populações. Maynard Smith e George R. Price estão entre os principais acadêmicos pioneiros na utilização da Teoria de Jogos para cenários biológicos. Em 1973, Maynard Smith e Price estenderam a Teoria de Jogos incorporando ferramentas da dinâmica de populações e, assim, dando origem a Teoria de Jogos Evolucionários [23, 24]. A Teoria de Jogos Evolucionários permitiu a descrição de comportamentos e estratégias mais sofisticadas, por meio de processos estocásticos, ou seja, possíveis sequências de decisões sujeitas a efeitos de memória. O estudo realizado pela zoologista Susan Riechert utilizando a teoria de M. Smith e G. Price em aranhas do tipo "Agelenopsis aperta", demonstrou que o comportamento destas, em disputas territoriais, depende fortemente da diferença de tamanhos corporal, e se a esta defendendo ou invadindo um território [10]. Com o sucesso desta nova abordagem, a Teoria de Jogos tornou-se uma cadeira importante dentro da biologia. Hoje em dia, vemos muitas aplicações da Teoria de Jogos Evolucionários sendo utilizadas por economistas e cientistas políticos [16, 25].

É comum, consideramos os jogadores como agentes racionais, ou seja, agentes que sempre adotam estratégias ótimas e que consideram seus adversários como igualmente racionais. Contudo, na natureza raramente observamos tal comportamento idealizado. Jogadores racionais não atingem um desempenho ótimo ao interagir com jogadores de racionalidade limitada, porque as estratégias ótimas assumem que os seus adversários são igualmente racionais. Outro argumento importante é que há 
sempre custos e riscos inerentes durante o processo de tomada de decisão. O formalismo dos Jogos Potenciais Evolucionários [26] captura estes fatores de racionalidade limitada e erros cometidos durante o processo de tomada de decisão. Lembramos que os Jogos Potenciais Evolucionários não é o único formalismo, "Trembling hand" e Bounded rationality são modelos que também consideram os erros e a racionalidade dos jogadores [27, 28]. Com os Jogos Potenciais Evolucionários, buscamos traçar um paralelo entre a Teoria de Jogos e a Mecânica Estatística, modelar o comportamento dos jogadores por meio de ferramentas da Mecânica Estatística. A abordagem estudada neste trabalho permite uma melhor compreensão de cenários nos quais ruídos, erros e a irracionalidade influenciam fortemente o processo de decisão dos jogadores.[18, 29-35]

\subsection{Dissertação}

Atualmente a Teoria de Jogos é uma teoria reconhecida e famosa pelas suas contribuições, aplicações e prêmios [36]. Por causa deste fatores, esta teoria é amplamente utilizada nas ciências Sociais e Políticas, Biologia, Economia e Computação. Teoria de Jogos demonstrou sucesso ao modelar comportamentos estratégicos e dilemas de cooperação assim como outras relações $[5,10,11,16]$.

Esse estudo surgiu de uma conversa entre O Ph.D professor Alexandre Souto Martinez, o aluno de pós doutorado Ph.D Gilberto de Medeiros Nakamura e o autor, Guilherme Contesini dos Santos. A prévia experiência do professor Alexandre em trabalhos acadêmicos na área da Teoria de Jogos e os trabalhos acadêmicos desenvolvidos pelo Laboratório de Modelagem de Sistemas Complexos (LMSC) permite que estudos como o apresentado nessa dissertação sejam conduzidos com destreza $[37-42]$. 


\subsection{Resultados e organização da Dissertação}

Essa dissertação tem como objetivo melhorar a compreensão e a descrição dos jogos e dos mecanismos que influenciam o processo de tomada de decisão. Estudamos nessa dissertação o "Jogo do Bem Público" com o formalismo dos Jogos Potenciais Evolucionários por meio de ferramentas da Termodinâmica e da Mecânica Estatística. Enunciamos o jogo utilizando a notação vetorial tradicional, fazendo uma análise sobre o dilema de cooperação. Mostramos que as funções que descrevem o ganho individual e coletivo podem ser expressas por meio da álgebra do $\mathfrak{s u}(2)$. A conexão entre os Jogos Potenciais e a Termodinâmica nos permite fazer uma analogia entre os jogos e sistemas magnéticos. Mostramos que o "Jogo do Bem Público" se comporta de modo análogo a uma cadeia de spin-1/2. Identificamos que o risco de cooperação é um fator intrínseco ao sistema necessário para descrever o comportamento dos jogadores previsto pelos resultados clássicos. Realizamos ensaios estatísticos para calcular a média e o desvio padrão das estratégias utilizadas. Identificamos que o inverso da frequência de erros cometidos durante o processo de decisão $(\beta)$ possui um papel fundamental para descrever um comportamento mais realístico dos jogadores. Realizamos simulações de Monte Carlo utilizando o algoritmo de Metropolis-Hastings e a dinâmica de Glauber, análogos a regra logit utilizada por Blume [43] para obter as soluções numéricas do problema, e que corroboram com os resultados analíticos [44].

A dissertação esta organizada da seguinte forma: no capítulo 2 seguinte, apresentamos os principais conceitos e fundamentos da Teoria de Jogos, exemplificandoos através de jogos de conhecimento comum. Ainda no mesmo capítulo, introduzimos conceito do equilíbrio de Nash e enunciamos o "Jogo do Bem Público". No capítulo 3, apresentamos a Teoria de Jogos Evolucionários, mostrando como a equação logística descreve a frequência de uso das estratégias e a descrição das Estratégias Reativas 
por meio de cadeias Marcovianas. No 4 capítulo, apresentamos o formalismo dos Jogos Potenciais Evolucionários e como ele se conecta com a Termodinâmica e a Mecânica Estatística. No capítulo 5, apresentamos os resultados analíticos e numéricos obtidos nesse trabalho. No último capítulo, debatemos as principais conclusões do trabalho. 


\section{2}

\section{A Teoria de Jogos}

Cada jogador deve aceitar as cartas que a vida lhe dá, mas uma vez elas em mão, ele deve decidir como jogar para ganhar o jogo.

- Voltaire

A Teoria de Jogos estuda o comportamento de agentes e suas decisões, fornecendo possíveis previsões sobre as decisões e comportamentos mais prováveis de ocorrer quando um ou mais agentes interagem. Neste capítulo, definimos os principais conceitos, terminologias e fundamentos da teoria de jogos, a serem utilizados nesse estudo. Fazemos a descrição destes fundamentos de modo instrutivo, exemplificando-os através do "jogo par ou ímpar"(JPI) [15]. Na sequência introduzimos o "Jogo do Bem Público" (JBP), cujo dilema de cooperação é o cerne desta dissertação.

\subsection{Fundamentos da Teoria de Jogos}

No capítulo anterior, vimos que a teoria de jogos é formada por uma família de modelos matemáticos chamados de jogos. O "jogo par ou ímpar", (JPI), comum ao conhecimento geral, é um exemplo de jogo. Todavia, relembramos aqui a sua descrição; no JPI, dois jogadores, ("jogador par" e "jogador ímpar"), devem escolher simultaneamente um número entre zero e dez. Em seguida, mostram com os dedos 
o número que representa a estratégia escolhida. Dizemos que o "jogador par" vence se a soma total dos números for um número par. Por consequência, dizemos que o jogador "ímpar" perde (se esse for o caso) [45]. O resultado simétrico ocorre quando a soma dos números resultar em um número ímpar. No caso em que ambos jogadores escolhem o número zero, haverá um empate, um cenário de menor interesse. Apesar de simples, o JPI ilustra os principais fundamentos da teoria de jogos.

A partir da descrição de um jogo, identificamos quatro ideias fundamentais (destacadas a seguir): dizemos que um jogo é um conjunto de regras nas quais jogadores adotam estratégias baseadas em preferências de modo a adquirir uma ganho $[13,18,26,45]$. Dentro da teoria de jogos, cada jogo é uma representação abstrata, uma analogia matemática a uma situação encontrada na natureza, de modo que, para cada tipo de jogo, existe um formalismo mais adequado. O JPI é uma analogia ao jogo par ou ímpar, comumente utilizado para decidir situações de impasse.

O Jogador é o primeiro conceito de um jogo. Definimos um jogador como um agente capaz de tomar decisões. Existem diversos exemplos de jogadores na natureza, principalmente na Biologia, aonde exemplos destes agentes incluem bactérias, células, tumores, aranhas, golfinhos, humanos, dentre muitos outros [1-7, 46]. Esta diversidade de jogadores com diferentes característica geram diferentes classificações para os jogos. Duas destas classes são importantes para esta dissertação, pois caracterizam se a cada rodada os jogadores podem, ou não, comunicar entre si antes de tomar uma decisão. Definimos um jogo como sendo jogo cooperativo, se é permitida a comunicação entre os jogadores, ou seja, se estes conhecem as estratégias dos demais jogadores antes do final da rodada. Esta comunicação entre os agentes possibilita o surgimento de comportamentos como acordos, coalizões, blefes e ameaças. Um exemplo clássico desta classe de jogos é o Pôquer, no qual os jogadores 
conhecem as estratégias dos seus adversários antes da "mesa" fechar a rodada. Em contrapartida, jogos não-cooperativos são os jogos nos quais a comunicação entre os jogadores não é permitida. O JPI é um exemplo desta classe, pois neste jogo, as decisões dos jogadores de cada rodada, são tomadas individualmente, simultaneamente. Nos jogos não-cooperativos, se um jogador souber previamente a estratégia do seu adversário, este jogador saberá com precisão qual a melhor estratégia deve usar para vencer.

O segundo conceito chave para a análise de um jogo são as estratégias, que inclusive dão nome a alguns jogos [45]. As estratégias são as representações de todas as possíveis decisões que os jogadores podem adotar. No $J P I$, temos onze possíveis estratégias, de zero à dez. Lembramos que apesar do JPI conter dois jogadores, jogos como o Pôquer podem envolver um número maior de jogadores.

Cada combinação das estratégias adotadas por cada jogador resulta em um desfecho do jogo. Definimos o payoff como o conjunto de todos os possíveis desfechos, juntamente com o rótulo associado à combinação de estratégias. Note que isto sugere que existe uma função payoff que mapeia um vetor contendo uma possível configuração de estratégias à um vetor de ganhos. Esta ideia de função será explorada no capítulos posteriores. Para o jogos com dois jogadores, é comum representar este conjunto de possíveis desfechos por meio da matriz de payoff. Podemos extrapolar e definir o payoff de um jogador como todos os possível ganhos que um jogador pode receber, se este escolher uma determinada estratégia, formando portanto um subconjunto do payoff. A definição do payoff feita aqui, é diferente da definição encontrada comumente na literatura, aonde o conceito de payoff e ganho se misturam.

Com os principais conceitos da Teoria de Jogos definidos, ainda temos dificuldade em compreender a motivação de um jogador em escolher uma determinada 
estratégia. Esta é uma das perguntas centrais na teoria de jogos alvo de inúmeros debates $[13,47,48]$. Na teoria de jogos, utiliza-se o princípio da utilidade, ou simplesmente utilidade para responder a motivação dos jogadores em escolher uma estratégia. O princípio da utilidade foi inicialmente introduzido por Bentham em 1789 [49], que argumenta:

Por "princípio da utilidade" entende-se o princípio que aprova ou desaprova toda ação de acordo com a tendência que aparenta ter de aumentar, ou diminuir - promover ou opor-se - à felicidade da pessoa ou grupo cujo interesse que está em questão. (...)

Por "utilidade" entende-se a propriedade de algo que tende a produzir benefício, vantagem, prazer, bem ou felicidade, ou evitar danos, dor, mal ou infelicidade de um grupo. (...)

É inútil falar do interesse da comunidade sem entender o interesse do indivíduo. Diz-se que uma coisa é "promover o interesse" de um indivíduo quando ele tende a aumentar a soma total de seus prazeres ou diminuir a soma total de suas dores.

(Bentham, An Introduction to the Principles of Morals and Legislation, page $[6-7]^{1}$ )

Bentham argumenta que um jogador sempre procura estratégias que lhe geram o maior benefício e que evitam danos. Este argumento nos permite identificar quais estratégias são mais prováveis de um jogador adotar. John von Neumann, um dos fundadores da Teoria de Jogos moderna, utilizou o princípio da utilidade de Bentham para relacionar a utilidade de um jogador com a estratégia mais provável de ser adotada [13]. Deste modo, a motivação dos jogadores está em buscar a estratégia que maximiza a sua utilidade, ou seja, aquela que maximiza o ganho e minimiza o risco. Apesar deste argumento ser importante, este é ainda simplório. Neumann, fazendo uma analogia com a Teoria do Calor, justifica que também é necessário um conjunto de preferências de modo a definir o espectro das estratégias.

Tudo isto é fortemente remanescente das condições existentes no início da teoria do calor: que também se baseava no conceito intuitivamente claro de sentir um corpo mais quente do que outro, ainda que

\footnotetext{
${ }^{1}$ Tradução feita pelo autor. Trecho de An Introduction to the Principles of Morals and Legislation - Chapter 1 - The Principle of Utility - 1780 - page [6-7][49]
} 
não houvesse modo imediato de expressar significativamente (quantitativamente) o quanto, ou quantas vezes.(...)

Essa comparação com o calor também mostra o quão pouco se pode prever a priori qual será a forma definitiva desta teoria. As indicações acima não revelam de modo algum o que, de fato, aconteceu posteriormente. Mostrou-se que o calor permite uma descrição quantitativa não por um número, mas por dois: a quantidade de calor e a temperatura. $(. .$.

O desenvolvimento histórico da Teoria do Calor indica que é preciso ser extremamente cuidadoso ao fazer negações sobre qualquer conceito com a pretensão de finalidade. Mesmo se as utilidades aparentam-se qualitativas hoje, a experiência histórica da Teoria do Calor pode se repetir, e ninguém pode predizer com quais ramificações e variações. E certamente não deveria desencorajar explicações teóricas das possibilidades formais de uma utilidade numérica.

(John von Neumann, Oskar Morgenstern - 1994 - Theory of Games and Economic Behavior - Chapter 3 - The Notion of Utility página 16-18)

2

Com as definição de utilidade e o conjunto de preferências, conseguimos organizar as estratégias de um jogador em um espectro. Dizemos que um jogador é racional, se baseado na sua utilidade e preferências), este adota a estratégia que maximiza o seu ganho final. Além disso, jogadores racionais também assumem que os seus adversários são igualmente racionais, e que estes adotam estratégias análogas. É importante acentuar que, a assunção de que os seus adversários são racionais influencia fortemente na escolha da melhor a estratégia. No exemplo do JPI, os possíveis ganhos são 1 para o vencedor, -1 para o perdedor e 0 no caso de empate. Dizemos que a preferência de cada jogador é vencer, ou seja, jogadores racionais procuram sempre obter 1. A tabela a seguir (2.1) é utilizada para representar o JPI.

\footnotetext{
${ }^{2}$ Tradução feita pelo autor. Trecho de Theory of Games and Economic Behavior - Chapter 3 - The Notion of Utility - page [16-18][13]
} 


\begin{tabular}{lrrr}
\hline Jogador 1\Jogador 2 & Par & Ímpar & Zero \\
\hline Par & $1,-1$ & $-1,1$ & $1,-1$ \\
\hline Ímpar & $-1,1$ & $1,-1$ & $-1,1$ \\
\hline Zero & $1,-1$ & $-1,1$ & 0,0 \\
\hline
\end{tabular}

Tabela 1: Representação Normal, no formato de tabela do JPI. A tabela de payoff mostra o que cada jogador recebe baseado nas possíveis estratégias adotadas. Em cada tupla, o primeiro número indica o ganho do jogador 1, enquanto o segundo número indica o ganho do segundo jogador, para cada payoff.

Duas classes de jogos emergem quando analisamos o payoff total dos jogadores. Elas são os jogos de soma-zero e jogos de soma variada (zero-sum games e non-zero-sum games). Como o próprio nome indica, os jogos de soma-zero são jogos cuja soma dos ganhos dos jogadores é nula. Esses jogos possuem a característica de serem estritamente competitivos, ou seja, não existe uma estratégia que resulte na cooperação entre os jogadores. Nos jogos de soma-zero, todo o ganho de um jogador é decorrência da perda do outro jogador. Se um jogador ganha, o outro obrigatoriamente perde. Em seu livro, Theory of Games and Economic Behavior, von Neumann e Morgenstern formulam o teorema minimax, o qual permite encontrar a solução ótima para os jogos desta classe [13]. O JPI é um exemplo de jogo de soma-zero, assim como o jogo pedra-papel-tesoura, o Pôquer e o xadrez. Na Biologia, a competição por acasalamento ou domínio territorial são exemplos dessa classe de jogos $[5,9-11]$.

Em contrapartida, nos jogos de soma variada a soma dos ganhos de todos os jogadores, não é exclusivamente zero, ou seja, pode assumir qualquer valor inclusive zero. Nesta classe de jogos existe uma variedade de comportamentos mais sofisticados, como a cooperação, coalizões, dentre outros. Os jogos de soma vari- 
ada permitem analogias com cenários da Biologia, como o comportamento de um formigueiro, crescimento de tumores, a pesca coletiva entre golfinho e pescadores, e muitos outros $[1-7]$.

O ganho de um jogador é resultado de um mecanismo intrincado. Os jogos de soma variada se destacam por capturar a natureza deste mecanismo. É nesta classe de jogos que dedicamos o nosso trabalho, pois são nestes jogos que os dilemas de cooperação emergem.

\subsection{Estratégias Dominantes e o Equilíbrio de Nash}

Argumentamos que jogadores racionais baseiam suas decisões conforme a suas preferências e possíveis ganhos. Contudo, o ganho de um jogador não depende somente da sua estratégia, mas da assunção do comportamento dos demais jogadores e da combinação das estratégias de todos os jogadores. Essa característica permite que a escolha de uma estratégia domine a decisão dos jogadores. Uma estratégia é tida dominante sobre outra estratégia quando, ao utilizar esta a estratégia, o jogador obtém o maior ganho possível, se o seu adversário permanecer com a mesma estratégia $[15,45]$. Dizemos a estratégia dominante é a melhor resposta à uma estratégia adversária. Representamos uma estratégia dominante por um vetor formado pelas estratégias utilizadas pelo jogador e o seu(s) adversário(s).

Dada a definição de estratégias dominantes, é interessante identificar os vetores de estratégias dominantes que simultaneamente maximizam o ganho e minimizam o risco. Em 1951, John F. Nash estendeu o conceito de estratégias dominantes para pontos de equilíbrio. Hoje chamamos de estes pontos de equilíbrio de Equilíbrio de Nash (EN). Quando os jogadores utilizam estratégias correspondente ao EN, eles não possuem estímulo em mudar de estratégias [20, 21]. Em uma analogia com um sistema físico, o $E N$ corresponde a um ponto de equilíbrio, porém não implica 
que o ponto de equilíbrio seja um mínimo global. No Equilíbrio de Nash, qualquer mudança isolada das estratégias dos jogadores retorna um ganho inferior ao jogador que decide mudar. É valido lembrar que o EN não necessariamente corresponde ao maior ganho global ou individual.

\subsection{O Jogo do Bem Público}

O objetivo desse estudo é investigar e contribuir com uma solução do dilema de cooperação chamado "tragédia dos comuns". Este é o dilema de cooperação que surge no "Jogo do Bem Público", JBP. Introduzimos, a seguir, o $J B P$ a fim de melhor compreender a natureza deste dilema. Considere a seguinte situação: uma comunidade com $\mathrm{N}$ moradores (jogadores) isolada por um rio. Decidi-se construir uma ponte, a fim de facilitar a travessia. Um possível acordo é proposto aos moradores desta comunidade, pois sabe-se que nenhum dos moradores possuem recursos suficientes para construir a ponte separadamente. Os jogadores então podem, aceitar o acordo (cooperar), pagando uma taxa $c$, parte do custo de construção da ponte "bem público", ou rejeitar o acordo (desertar), evitando este custo. A decisão de cada morador é individual e simultânea, ou seja, não pode haver coalizão, blefes, represália, ou qualquer tipo de comunicação entre os moradores. Após todos os jogadores terem tomado uma decisão, o montante arecadado é acrescido por um juro, e o montante final é dividido igualmente entre todos os jogadores, incluindo aqueles que não cooperaram. Podemos imaginar que, se o valor do juro aplicado for muito grande, apenas um cooperador será necessário para que todos maximizem o seu ganho final. Por outro lado, se o juros for inferior a unidade, o montante final será inferior ao montante arecadado, e consequentemente nenhum jogador terá incentivo para cooperar. Portanto, o juro aplicado $r$ ao montante possui valores restritos, definidos pela relação $(1<r<N-1)$ [19, 50-53]. Observe que esta condição impõe 
ao JBP um número mínimo de três jogadores ${ }^{3}$. A divisão do montante entre todos os jogadores simboliza que todos os moradores são beneficiados igualmente com a construção da ponte, do bem público, incluindo aqueles que não contribuíram com o custo da construção.

No $J B P$, vemos o surgimento de um dilema de cooperação. O dilema emerge quando um morador, (racional), percebe que consegue maximizar o seu lucro individual ao desertar. Como consideramos todos os moradores como agentes racionais, todos irão desertar e a ponte não será construída, resultando no pior cenário possível. Esse resultado difere dos experimentos observados na natureza, onde os agentes optam por cooperar no maior número dos cenários [54]. Portanto, observamos uma contradição entre o resultado do modelo e o observado na natureza, ou seja, um dilema de cooperação. No $J B P$, este dilema de cooperação é conhecido como "a tragédia dos comuns" (Tragedy of the commons) [19]. Esse jogo captura com primor o dilema de cooperação que ocorre em um grupo de jogadores, no qual o comportamento individual ótimo difere do ótimo comportamento global.

\footnotetext{
${ }^{3}$ É comum encontrar na literatura, autores afirmando que o número mínimo de jogadores para o $J B P$ é dois, e que nesta situação o jogo se reduz ao "dilema do Prisioneiro". Discordamos desta análise, por que no caso em que há apenas dois jogadores o único valor possível para o juro será $r=1$. É fácil ver que cenário todo o montante arrecado será o montante distribuído, e o jogo se reduz a um jogo de "soma-nula", que possui solução pelo teorema $\min \max$.
} 


\title{
3
}

\section{A Teoria de Jogos Evolucionários}

\author{
A matemática compara os mais diversos fenômenos e descobre as \\ analogias secretas que os unem.
}

- Jean Baptiste Joseph Fourier

A descrição dos cenários por meio de jogos é útil, porém muitos dos comportamentos dos animais são adquiridos ao longo de inúmeras iterações. Os experimentos de Pavlov são exemplos clássicos que verificam essa asserção [55]. Os jogos com múltiplas rodadas, ou seja, iterados são uma evolução natural dos jogos clássicos. Essa classe de jogos permite uma descrição mais realista do processo de tomada de decisão encontrados na natureza, e possibilitam o estudo dos mecanismos que promovem comportamentos distintos. Efeitos de memória, reputação, aplicação de punição, recompensa, e comportamentos como a seleção de parentesco (kin selection), dentre outros são exemplos destes mecanismo. Os formalismos mais utilizados para estudar essa classe de jogos são a Teoria de Jogos Evolucionários (TJE), introduzida por Maynard Smith e Price [23], abordagem por meio dos Jogos de Estratégias Evolutivas feita por Martin Nowak, Karl Sigmund e Robert May [56-59] e a teoria dos Jogos Potenciais Evolucionários (JPE), introduzido por Blume e ampliado por [18, 26, 29, 31, 34, 43, 44, 60-64]. Neste capítulo revisamos a A Teoria de Jogos Evolucionários e os Jogos de Estratégias Evolutivas. 


\subsection{Teoria de Jogos Evolucionários}

A Teoria de Jogos Evolucionários TJE é o formalismo que utiliza dinâmicas evolutivas para descrever a dinâmica da frequência das estratégias. Essa teoria foi introduzida por John Maynard Smith e George Price [14, 65]. Para ilustrar este formalismo considere um jogo descrito pela matriz de payoff (3.1), com 2 jogadores e duas estratégias, (3.2). Denotamos $\rho_{1}$ e $\rho_{2}$, como as frequências de uso destas estratégias pelos jogadores $\left\{\rho_{1}, \rho_{2} \in \mathcal{R} / \rho_{1}, \rho_{2} \in[1,0]\right\}$. A frequência de uma estrategia é, então, o número de jogadores que utilizam a estratégia $s_{1}$, dividido pelo número total de jogadores. Maynard Smith e Price fazem uma analogia entre essa frequência estratégia e a densidade populacional de uma espécie, utilizando deste modo a equação replicadora, também conhecida como mapa logístico [57, 58, 65].

$$
V=\left(\begin{array}{ll}
a & b \\
c & d
\end{array}\right)
$$

Representação matricial de um jogo genérico com duas estratégias.

$$
s_{1}=\left(\begin{array}{l}
1 \\
0
\end{array}\right) \quad, \quad s_{2}=\left(\begin{array}{l}
0 \\
1
\end{array}\right)
$$

Dada uma matriz de payoff, associamos o "fitness" de cada estratégia ao ganho médio (3.3), tal que, a equação (3.4) modela a dinâmica da frequência $\rho_{1}$. Como nesse jogo existem somente duas estratégias, utilizamos o vínculo para determinar a dinâmica de ambas estratégias em termos de apenas uma das densidades (3.5). Por 
fim reescrevemos a evolução temporal de $\rho_{1}$ como (3.7).

$$
\begin{gathered}
\left\langle V_{i}\right\rangle=\sum_{i=1}^{2} V_{i j} s_{j} s_{i} \\
\frac{d}{d t} \rho_{1}=\rho_{1}\left[\rho_{1}\left\langle V_{1}\right\rangle-\langle V\rangle\right] \\
\rho_{1}+\rho_{2}=1 \\
\langle V\rangle=\sum_{j=1}^{2} \rho_{j}\left\langle V_{j}\right\rangle \\
\frac{d}{d t} \rho_{1}=\rho_{1}\left(1-\rho_{1}\right)\left(\left\langle V_{1}\right\rangle-\left\langle V_{2}\right\rangle\right) \\
V_{2}=c \rho_{1}+d \rho_{2} \\
V_{1}=a \rho_{1}+b \rho_{2}
\end{gathered}
$$

Com a equação (3.7), observamos que frequência que possui o maior "fitness" médio $\left\langle V_{j}\right\rangle$ será a mais utilizada pelos jogadores, $\left(\rho_{j} \rightarrow 1\right)$. Note que a diferença entre a média dos payoffs $\left\langle V_{1}\right\rangle-\left\langle V_{2}\right\rangle$ é equivalente ao parâmetro de controle no mapa logístico com tempo discreto, e que, se essa diferença for suficientemente grande $\left(\left\langle V_{1}\right\rangle-\left\langle V_{2}\right\rangle\right) \in[3.57,4]$ haverá a emergência de fenômenos caóticos [66], ao decorrer das rodadas ${ }^{1}$.

A análise de máximos e mínimos da equação replicadora e calculando o payoff médio de cada estratégia como (3.8) e (3.9) expõe os pontos de equilíbrio existentes

\footnotetext{
${ }^{1}$ Como esse formalismo não é cerne dessa dissertação não consideramos esses efeitos. Para uma análise mais detalhada sobre este formalismo recomentamos os textos $[57,66,67]$
} 
para esse jogo. Desta análise concluímos que se o ganho médio dos jogadores que utilizam a estratégia $s_{1},(3.3)$ for maior que o ganho médio de $s_{2},\left(\left\langle V_{1}\right\rangle-\left\langle V_{2}\right\rangle>0\right.$, então, $s_{1}$ será a estratégia dominante de modo que no limite para um número grande rodadas $\left(\lim _{t \rightarrow \infty} \rho_{1} \rightarrow 1\right)$. No caso contrário $\left(\left\langle V_{1}\right\rangle-\left\langle V_{2}\right\rangle\right)<0$ a frequência irá diminuir até atingir outro ponto de equilíbrio $\rho_{1}=0$. Para $\left(\left\langle V_{1}\right\rangle-\left\langle V_{2}\right\rangle\right)=0$, temos um terceiro cenário que permite a coexistência entre as estratégias, o ponto de equilíbrio e a sua estabilidade depende da relação dos parâmetros da matriz de payoff. Utilizando (3.8), (3.9) e $V_{1}=V_{2}$, calculamos o ponto de equilíbrio $\rho^{*}$, como (3.10) $\left\{\rho^{*} \subset[0,1]\right\}$.

$$
\rho^{*}=\frac{d-b}{a+d-c-b}
$$

$\mathrm{Na} T J E$, associamos as estratégias que atingem os pontos de equilíbrio ao Equilíbrio de Nash. Nesse formalismo utiliza-se a premissa de racionalidade ótima, na qual todos os jogadores não cometem erros e não desviam da estratégia ótima. Contudo, como já discutido nos capítulos anteriores, essa premissa não é muito realista quando comparamos com observações e experimentos. Apesar deste fato, a TJE obtém resultados importantes, e revela a existência de um ponto de coexistência de estratégias $\rho^{*}$. Este ponto de coexistência explica o porque não observamos somente estratégias dominantes na natureza, e serve como uma primeira resposta para os dilemas de cooperação. Por fim, concluímos que a TJE fornece uma rica descrição sobre a dinâmica das estratégias para os jogos iterados.

\subsection{Estratégias Evolutivas}

Estratégias Evolutivas é uma descrição que busca um comportamento mais próximo ao do observado na natureza. Uma Estratégia evolutiva é um comportamento dos jogadores que captura os efeitos da última rodada, ou seja, uma estratégia 
que considera a memória dos jogadores. Para ilustrar este formalismo considere um jogo (3.1) com dois jogadores e duas estratégias puras (3.2), note que as estratégias puras são agora interpretadas como estados dos jogadores. Uma estratégia evolutiva é descrita por dois parâmetros $(p, q)$, interpretados como probabilidades, e são definidos como: a probabilidade de cooperar dado que o seu oponente cooperou na última rodada $\left(p_{1}\right)$ e a probabilidade de cooperar dado que o seu oponente desertou na última rodada $\left(q_{1}\right)[56,57]$. Para o segundo jogador os parâmetros $p_{2}$ e $q_{2}$ descrevem as mesmas probabilidades. Alguns exemplos famosos de estratégias evolutivas são: a estratégia aleatória $\operatorname{RANDOM}(1 / 2,1 / 2)$; o "Tit-For-Tat" TFT $(1,0)$; o "Generous Tit-For-Tat" GTFT (1,1/3); a estratégia de sempre cooperar "Always cooperates" $A L L C(1,1)$ e a estratégia de sempre desertar "Always defect" ALLD $(0,0)[38]$.

Para este jogo iterado utilizamos uma cadeia Marcoviana (3.11) para descrever a dinâmica das estratégias puras. Uma cadeia Marcoviana é um processo estocástico que descreve uma sequência de prováveis estados $\mu(t+1)$, com base no estado anterior $\mu(t)$ e em uma matriz de transferência $T(\mu(t) \mid \mu(t+1))$. A evolução temporal dos estados dos jogadores é dada por essa matriz transição (3.12), e os estados do jogo são as combinações dos possíveis estados de cada jogador, ou seja, combinações das estratégias puras $s_{1} \otimes s_{2}[56,57]$. Estas combinações formam um espaço vetorial com quatro estados, descritos por (3.13) e (3.14).

$$
\begin{gathered}
\mu(t+1)=T[\mu(t) \rightarrow \mu(t+1)] \cdot \mu(t) . \\
W=\left(\begin{array}{llll}
p_{1} p_{2} & q_{1} p_{2} & p_{1} q_{2} & q_{1} q_{2} \\
p_{1}\left(1-p_{2}\right) & q_{1}\left(1-p_{2}\right) & p_{1}\left(1-q_{2}\right) & q_{1}\left(1-q_{2}\right) \\
\left(1-p_{1}\right) p_{2} & \left(1-q_{1}\right) p_{2} & \left(1-p_{1}\right) q_{2} & \left(1-q_{1}\right) q_{2} \\
\left(1-p_{1}\right)\left(1-p_{2}\right) & \left(1-q_{1}\right)\left(1-p_{2}\right) & \left(1-p_{1}\right)\left(1-q_{2}\right) & \left(1-q_{1}\right)\left(1-q_{2}\right)
\end{array}\right)
\end{gathered}
$$


Representação matricial de um jogo genérico com duas estratégias evolutivas.

$$
\begin{aligned}
& \mu_{c c}=\left(\begin{array}{l}
1 \\
0 \\
0 \\
0
\end{array}\right), \mu_{c d}=\left(\begin{array}{l}
0 \\
1 \\
0 \\
0
\end{array}\right) \\
& \mu_{d c}=\left(\begin{array}{l}
0 \\
0 \\
1 \\
0
\end{array}\right), \mu_{d d}=\left(\begin{array}{l}
0 \\
0 \\
0 \\
1
\end{array}\right)
\end{aligned}
$$

Como a matriz de transição (3.12) descreve a evolução do estado $\mu_{t}$, calculamos o estado de estacionário, por meio da repetidas aplicações da matriz de transferência (3.11). Pelo teorema de Perron-Frobenius, se a matriz de transferência for estocástica e positiva, ou seja, se a soma dos elementos de cada coluna for igual a um e se todos os elementos forem positivos, então essa matriz possui o maior autovalor igual a um, e o estado de estacionário do sistema será o autoestado associado a esse autovalor. Portanto, o estado estacionário e o ganho total do jogo $\left(p_{1}, q_{1}\right),\left(p_{2}, q_{2}\right)$, são calculados pelas relações (3.15) e (3.16),

$$
E\left(\left(p_{1}, q_{1}\right),\left(p_{2}, q_{2}\right)\right)=a m_{1} m_{2}+b m_{1}\left(1-m_{2}\right)+c\left(1-m_{1}\right) m_{2}+d\left(1-m_{2}\right)
$$


Para as estratégias evolutivas o equilíbrio de Nash é associado às estratégias evolutivas que maximizam o ganho dos jogadores, logo, o EN é o par de estratégias evolutivas $\left(p^{*}, q^{*}\right)$ que maximiza o ganho para o jogador.

O cientista político Robert Axelrod realizou um torneio entre estratégias evolutivas para determinar a melhor [68]. Estas estratégias foram testadas no jogo do dilema do prisioneiro, na qual a estratégia "Tit-for-Tat"(TFT) obteve o melhor desempenho, quando testada contra as estratégias submetidas. Essa estrategia é está associada ao comportamento de reciprocidade ao cooperador e retalhação ao desertor. Ao utilizar o TFT, os jogadores cooperam somente se o seu adversário cooperou na rodada anterior e deserta no caso contrário. Os jogadores que utilizaram o TFT, obtiveram um bom desempenho quando enfrentam jogadores que sempre desertam $A L L D$, ou sempre cooperavam $A L L C$. Muitas das estratégias evolutivas são simuladas por meio de Automata Cellular, o que permite o estudo dos efeitos que estruturas espaciais dos jogadores tem sobre o resultado do jogo [67]. Lembramos que no formalismo das estratégias evolutivas a condição inicial afeta significativamente o resultado do jogo. Esta limitação é motivo de estudar um formalismo mais geral, que não dependa de número grande de parâmetros, nem das condições iniciais do problema, e que seja estável a erros. 


\title{
4
}

\section{Jogos Potenciais Evolucionários}

\author{
Reserve o seu direito de pensar, pois até mesmo o pensamento er- \\ rado é melhor do que não pensar em nada.
}

- Hypatia

Os Jogos Potenciais Evolucionários é um formalismo dentro da Teoria de Jogos que recebeu contribuições significativas nos últimos anos, devido a sua conexão com a Mecânica Estatística. Neste capítulo, introduzimos este formalismo, definindo as principais propriedades, analisando como é feita a conexão com a Mecânica Estatística e determinando quais são as ferramentas que podem ser utilizadas para análise e caracterização dos jogos.

\subsection{Jogos potenciais}

O Jogo Potencias Evolucionário (JPE) é um formalismo utilizado para estudar os jogos iterados. Um JPE é uma abordagem equivalente às regras evolutivas da Teoria de Jogos Evolucionários as quais, definem como cada jogador adota uma estratégia por rodada. Essas abordagem se baseia em um processo estocástico descrito por meio de uma cadeia Marcoviana. Em contraste, os JPE permitem caracterizar o sistema no equilíbrio após inúmeras rodadas usando de ferramentas da Termodinâmica e da Física Estatística. 
Exemplificamos os principais conceitos dos jogos potenciais por meio de um jogo simples, entre dois jogadores $(A, B)$, com duas estratégias $\left(n, n^{*}\right)$, uma função payoff $(u)$ que mapeia as combinação de estratégias em oito distintos ganhos $\left(u_{A}\left(n_{A}, n_{B}\right), u_{A}\left(n_{A}^{*}, n_{B}\right), \ldots\right.$, $\left.u_{B}\left(n_{A}, n_{B}^{*}\right), u_{B}\left(n_{A}^{*}, n_{B}^{*}\right)\right)$. Utilizamos a notação $u_{A}\left(n_{A}, n_{B}^{*}\right)$ para indicar o ganho que o jogador $A$ recebe quando este adota a estratégia $s$, visto que seu adversário $B$ adota a estratégia $n^{*}$. Dizemos que um jogo é potencial quando um jogador, (e.g. A) ao transicionar entre estratégias (e.g. $n_{A} \rightarrow n_{A}^{*} \rightarrow n_{A}$ ), formando um ciclo fechado, tem a soma das diferenças entre os seus ganhos igual a zero. Se o jogo simples for potencial, então a seguinte relação é considerada verdadeira,

$$
u_{A}\left(n_{A}, n_{B}\right)-u_{A}\left(n_{A}^{*}, n_{B}\right)-\left[u_{A}\left(n_{A}^{*}, n_{B}\right)-u_{A}\left(n_{A}, n_{B}\right)\right]=0 .
$$

Durante a transição de estratégias, é suficiente e necessário: que uma só mudança de estratégia ocorra por vez (4.2); que os jogadores retornem ao conjunto de estratégias inicial, satisfazendo (4.1); e que a diferença entres os ganhos independa do jogador (4.3), garantindo jogadores simétricos [18, 26, 44, 60, 61],

$$
\begin{gathered}
u_{A}\left(n_{A}, n_{B}\right) \rightarrow u_{A}\left(n_{A}^{*}, n_{B}\right) \rightarrow u_{A}\left(n_{A}^{*}, n_{B}^{*}\right) \rightarrow u_{A}\left(n_{A}, n_{B}^{*}\right) \rightarrow u_{A}\left(n_{A}, n_{B}\right) . \\
u_{A}\left(n_{A}, n_{B}\right)-u_{A}\left(n_{A}^{*}, n_{B}\right)=u_{B}\left(n_{B}, n_{A}\right)-u_{B}\left(n_{B}^{*}, n_{A}\right) .
\end{gathered}
$$

Se um jogo satisfizer estas condições, então, a função payoff será uma função potencial, $V\left(n_{A}, n_{B}\right)$, que mapeia a diferença entre os ganhos obtidos durante cada transição entre estratégias. Dizemos que $V\left(n_{A}, n_{B}\right)$ é uma função potencial que 
mapeia em um número as diferenças entre os ganhos,

$$
u_{A}\left(n_{A}, n_{B}\right)-u_{A}\left(n_{A}^{*}, n_{B}\right)=V\left(n_{A}, n_{B}\right)-V\left(n_{A}^{*}, n_{B}\right) .
$$

Fixamos um ganho em particular $\left.\left(V\left(n_{A}^{*}, n_{B}^{*}\right) \equiv 0\right)\right)$ para construir uma matriz potencial de payoff, uma representação equivalente à matriz de payoff. A matriz (4.5) é um exemplo da matriz potencial de payoff do jogo simples.

\begin{tabular}{lrr}
\hline$A \backslash B$ & $n_{B}$ & $n_{B}^{*}$ \\
\hline$n_{A}$ & $V\left(n_{A}, n_{B}\right)$ & $V\left(n_{A}, n_{B}^{*}\right)$ \\
\hline$n_{A}^{*}$ & $V\left(n_{A}^{*}, n_{B}\right)$ & 0 \\
\hline
\end{tabular}

Tabela 2: Matriz potencial de payoff para o jogo simples. Observe que, quando ambos os jogadores utilizam a estrategia $n^{*}$, o ganho resultante é definido como zero, $\left(V\left(n_{A}, n_{B}^{*}\right) \equiv 0\right)$.

Apesar dos JPE imporem restrições, neste formalismo conseguimos descrever a dinâmica de um jogo iterado utilizando a função potencial. Entre os jogos que podem ser descritos por este formalismo estão o "Jogo do Bem Público", o "Dilema do Prisoneiro", a "Caça ao Alce", "Jogo da Galinha", "Batalha dos Sexos", entre outros. Estudamos aqui o jogo do Bem Publico seguindo uma abordagem semelhante a feita pelas referências [18, 26, 31, 44, 60, 61].

\subsection{Conexão entre estratégias estocásticas e a termodinâ- mica}

Em um jogo de estratégias estocásticas, dois jogadores tem as suas estratégias representadas por estados que evoluem para um estado estacionário $\mu$. O objetivo está em explorar diferentes estratégias. Associamos o EN à estratégia estocástica que proporciona o maior ganho aos jogadores $[57,59,67,69,70]$. Como apresentado 
no capítulo anterior, o cálculo para determinar a melhor estratégia estocástica pode ser feito por meio do teorema Perron-Frobenius e por meio de simulações de Cellular Automaton. Nos estudos realizados por Blume, mostrou-se uma abordagem alternativa por meio da equação mestra. Podemos descrever a evolução temporal da probabilidade de encontrar o sistema em uma dada configuração $P(\mu, t)$ por meio da equação equação mestra, com as taxas de transição $W\left(\mu \rightarrow \mu^{*}\right)$ e $W\left(\mu^{*} \rightarrow \mu\right)$, (4.6) [71],

$$
\frac{d}{d t} P(\mu, t+1)=\sum_{\mu^{*}}^{N}\left[W\left(\mu^{*} \rightarrow \mu\right) P\left(\mu^{*}, t\right)-W\left(\mu \rightarrow \mu^{*}\right) P(\mu, t)\right]
$$

Blume mostrou que esse cálculo também pode ser feito por meio de uma regra "logit" (4.7), que diz que um jogador irá mudar de estratégia, se esta aumentar o seu ganho individual. A regra "logit" descreve então a taxa de transição entre os estados $W\left(\mu \rightarrow \mu^{*}\right)$, [43, 64]. A correspondência entre os jogos potenciais e a termodinâmica é feita comparando a regra "logit" com um sistema termodinâmico no equilíbrio. Na física esta condição expressa pela regra "logit" é conhecida como "dinâmica de Glauber", a qual satisfaz o balanço detalhado (4.8),

$$
\begin{gathered}
W\left(\mu \rightarrow \mu^{*}\right)=\frac{1}{1+\mathrm{e}^{-\beta\left[u\left(\mu^{*}\right)-u(\mu)\right]}} \\
W\left(\mu \rightarrow \mu^{*}\right) P(\mu)=W\left(\mu^{*} \rightarrow \mu\right) P\left(\mu^{*}\right)
\end{gathered}
$$

Se a condição do balanço detalhado for satisfeita, o sistema no equilíbrio será descrito pela distribuição de Boltzmann (4.9) [72-75], permitindo uma descrição estatística do sistema. Nesta abordagem interpretamos a priori a função potencial $E(\mu)$ como a função payoff de cada jogador ${ }^{1}$, e o valor de $\beta$ como o erro médio

\footnotetext{
${ }^{1}$ Note que se o jogadores forem idênticos a função payoff será única
} 
cometido pelos jogadores ao adotar uma estratégia. Portanto, identificamos a função potencial como uma função proporcional ao negativo da "Energia" dos sistemas e $\beta$ proporcional ao inverso da temperatura.

Sabendo que o sistema é descrito por uma distribuição de Boltzmann, o problema se resume à um problema da mecânica estatística, mais precisamente ao de um sistema em "banho térmico" ${ }^{2}$. Para resolver um problema deste tipo calculamos a função partição $(4.9)(Z)^{3}$. Esta função consiste em uma soma, ponderada por $\beta$, sobre todos os possíveis microestados de energia do sistema, ou seja, é uma função que auxilia no calculo da distribuição de probabilidades do sistema [76]. Blume concluiu que a estratégia média e o ganho médio de um jogo podem ser calculadas por meio da função de partição do sistema.

$$
P\left(n_{A}, n_{B}\right)=\frac{1}{Z} \sum_{\mu} e^{\beta E(\mu)} \quad, \quad Z=\sum_{\mu} e^{-\beta\left(E\left(\mu^{*}\right)-E(\mu)\right)}
$$

Resumimos este processo da seguinte forma: Na abordagem tradicional, é utilizada uma Cadeia Marcoviana cuja a taxa de transição é definida pelas estratégias estocásticas. Na abordagem proposta por Blume, ao invés de estudar o espaço de estratégias estocásticas, utilizamos a equação mestra (4.6) juntamente com a regra "logit", para descrever a evolução temporal da probabilidade de encontrar o sistema em um determinado estado. Como a regra "logit” equivale à dinâmica de Glauber, então balanço detalhado é satisfeito, e o sistema no equilíbrio será descrito pela distribuição de Boltzmann, da qual a média das estratégias adotadas pode ser obtida. Esse resultado é o núcleo do nosso estudo, pois essa abordagem permite o estudo dos mecanismos que promovem a cooperação entre jogadores por meio das ferramentas

\footnotetext{
${ }^{2} \mathrm{O}$ "banho térmico" é um jargão muito utilizado dentro da mecânica estatística para designar um sistema em contato com um reservatório de calor à temperatura constante

${ }^{3}$ Este é um procedimento regularmente utilizado mecânica estatística para se calcular as propriedades de um sistema em "banho térmico" somente por meio da energia potencial do sistema
} 
da Mecânica Estatística.

O estudo das Simulações de Monte Carlo $(S M C)$ nos ajudam na interpretação da energia, $\left(E\left(\mu^{*}\right)-E(\mu)\right)$. As $S M C$ utilizam as mesmas Cadeias Marcovianas, que foram anteriormente apresentadas. Nessas simulações, dinâmicas fictícias equivalem à taxas de transição entre estratégias $W^{*}\left(\mu \rightarrow \mu^{*}\right)$, utilizada para obter uma sequência de estados. Inspirado nas dinâmicas de Glauber (4.10) [72] e de Metropolis-Hasting $^{4}$ (4.11) [75], identificamos novamente o paralelo entre função potencial e a energia potencial de um sistema físico. Definimos agora a energia como $\Delta E=\left(E\left(\mu^{*}\right)-E(\mu)\right)=-\left(V\left(\mu^{*}\right)-V(\mu)\right)$, tal que, $\mu$ e $\mu^{*}$ denotam diferentes configurações de estratégias. Dizemos que na teoria de jogos, quando os jogadores escolhem maximizar o seu ganho, equivale a dizer que um sistema físico tende à minimizar a sua energia.

$$
\begin{gathered}
P(\Delta E, \beta)=\frac{1}{1+e^{-\beta\left(E\left(\mu^{*}\right)-E(\mu)\right)}} \\
P(\Delta E, \beta)=e^{-\beta\left(E\left(\mu^{*}\right)-E(\mu)\right)}
\end{gathered}
$$

A dinâmica de Glauber (4.10), e separadamente a de Metropolis-Hasting (4.11) é uma condição imposta que define a probabilidade do sistema em aceitar, ou não, uma mudança de estratégia [73]. O procedimento é feito por meio de uma simulação, na qual a estratégia de cada jogador é representada por um número, 1 para cooperação, ou 0 para deserção. A lógica das dinâmicas consiste em; propor um mudança de estratégia ${ }^{5}$; calcular a diferença na energia global $\Delta E$ causada pela possível mudança; gerar um número pseudo-aleatório uniforme; se $\Delta E$ for negativa a

\footnotetext{
${ }^{4} \mathrm{~A}$ dinâmica Metropolis-Hasting é equivalente à dinâmica de Glauber, pois ambas são dinâmicas que atualizam um único componente por passo de Monte Carlo e levam ao equilíbrio térmico. A diferença entre as duas dinâmicas está na diferença entre os transientes, contudo como o na teoria dos jogos potenciais não nos preocupamos com o transiente, ambas as dinâmicas são equivalentes.

${ }^{5}$ Este procedimento é chamado de Passo Monte Carlo
} 
mudança é realizada, no caso em que $\Delta E$ for positivo, se o número pseudo-aleatório for menor que a relação (4.10), ou (4.11), então a mudança é realizada, caso contrário a mudança é rejeitada; Se a simulação seguir uma destas dinâmicas, é garantido obter os estados do sistema no equilíbrio ao término da simulação.

A interpretação de $\beta$ não é tão direta como a interpretação da energia. Como havíamos mencionado nos capítulos (1) e (2), os jogadores adotam estratégias baseando-se nos payoff e nas preferências. Contudo, observamos na natureza, situações nas quais alguns jogadores não seguem estritamente este argumento. Um modo de compreender estas situações é dizer que estes jogadores cometem erros quando adotam estratégias. Ao introduzimos $\beta$, quantificamos este comportamento, ou seja, $\beta$ é proporcional ao inverso da quantidade média de erros que os jogadores cometem durante o processo de tomada de decisão. Em Termodinâmica, $\beta$ é proporcional ao inverso da temperatura. Desde modo, $\beta$ é uma variável estritamente positiva, tal que, quando $\beta \rightarrow \infty$ corresponde aos cenários com jogadores estritamente racionais, em outras palavras, jogadores que nunca cometem erros. Por outro lado, quando $\beta \rightarrow 0$, vemos cenários nos quais os jogadores cometem muitos erros, jogadores que não utilizam uma regra para adotar uma estratégia e adotam estratégias aleatoriamente, ignorando as suas preferências e payoff.

Nos estudos realizados $[18,26,31,60,61]$, os autores fornecem algumas possíveis interpretações para significado de $K \equiv \beta^{-1}$. Nesses estudos, K pode representar; a irracionalidade dos jogadores; os erros cometidos pelos jogadores durante o processo de tomada de decisão; ou uma possível flutuação do ganho dos jogadores. Estes autores também argumentam que em sistemas sociais, esta variável pode ser possivelmente uma característica social do grupo.

Então, com isso, pudemos observar que nos jogos potenciais evolucionários não é necessário definir uma estratégia estocástica que depende de dois parâmetros 
arbitrários. Mostramos que o sistema no equilíbrio é descrito pela distribuição de Boltzmann e equivale ao EN. A função potencial é equivalente à energia de um sistema termodinâmico, $\left(E\left(\mu^{*}\right)-E(\mu)\right)=-\left(V\left(\mu^{*}\right)-V(\mu)\right)$, e $\beta$, apesar não possuir uma interpretação precisa, relacionada ao ruído do sistema. Este resultado nos assegura a caracterização do sistema através de ferramentas da Mecânica Estatística e da Termodinâmica. Mostramos que os JPE permitem uma descrição macroscópica dos jogos iterados, ao invés da descrição microscópica tradicional, observando o comportamento médio dos jogadores, rodada a rodada [18, 26, 44, 60, 61]. 


\section{Resultados}

A física tem uma história de sintetizar muitos fenômenos em poucas teorias.

- Richard P. Feynman

O tema desse projeto é estudar a conexão entre a Termodinâmica e a Teoria de Jogos. Nos capítulos anteriores, identificamos condições nas quais, as ferramentas da Mecânica Estatística e da Termodinâmica podem ser utilizadas para determinar a dinâmica dos jogos com dilemas de cooperação. O ponto principal deste estudo é, portanto, aplicar as ferramentas da Mecânica Estatística para o Jogo do Bem Público (JBP), e estudar como o mecanismo de punição, afeta a dinâmica do jogo. Comparamos os conceitos de estratégia, payoff e Equilíbrio de Nash $(E N)$ com os elementos de um sistema magnético. Neste capítulo, apresentamos os resultados obtidos nesse estudo, identificando a equivalência entre o $J B P$ com um sistema magnético.

\subsection{Notação algébrica para os jogos potenciais evolutivos}

A notação clássica utilizada nos $J P E$ representa as estratégias dos jogadores por vetores, e a função potencial por uma matriz. Para exemplificar esta notação, considere um jogo com dois jogadores $A$ e $B$ e duas estratégias ( $n$ e $\left.n^{*}\right)$, tais que, 


$$
n=\left(\begin{array}{l}
1 \\
0
\end{array}\right) \quad, \quad n^{*}=\left(\begin{array}{l}
0 \\
1
\end{array}\right)
$$

Representamos o jogador $A$ escolhendo a estratégia $n$ como $n_{A}$, e seu respectivo payoff como $\left(n_{A}\right)^{T} \cdot V_{A} \cdot n_{B}$, visto que o jogador $B$ adota a estratégia $n_{B}$. Observe que nesta notação podem ocorrer equívocos quanto a definição da matriz de payoff. Sabendo que, $\left(n_{B}\right)^{T} \cdot V_{A} \cdot n_{A} \neq\left(n_{A}\right)^{T} \cdot V_{A} \cdot n_{B} \equiv\left(n_{B}\right)^{T} \cdot\left(V_{A}\right)^{T} \cdot n_{A}$, é necessário definir a ordem da multiplicação da matriz de payoff.

Neste trabalho propomos uma notação equivalente, baseada em operadores e autovetores e que evita equívocos. Nesta nova notação, as estratégias são representadas pelo produto tensorial dos vetores de estratégias, $n_{A} \otimes n_{B} \equiv\left|n_{A} n_{B}\right\rangle$, autoestados do operador payoff $\hat{\varepsilon}_{A}$. Note que alterar a ordem dos jogadores equivale a alterar os índices. Utilizamos o operador número $\hat{n}$, da álgebra do $\mathfrak{s u}(2)$ para descrever jogos com duas estratégias. Os autovalores do operador número são as próprias estratégias $\left\{n, n^{*}\right\}, \hat{n}_{A}\left|n_{A} n_{B}\right\rangle=n_{a}\left|n_{A} n_{B}\right\rangle$,

$$
\hat{n}=\left(\begin{array}{ll}
1 & 0 \\
0 & 0
\end{array}\right)
$$

Representação matricial do operador número na álgebra $\mathfrak{s u}(2)$.

Para jogos com mais de dois jogadores, vemos que a notação matricial não é possível, por outro lado a notação de operadores permanece a mesma. Para jogos como múltiplos jogadores e duas estratégias, os autovalores do operador número permanecem os mesmos, $\hat{n}_{A}\left|n_{A} n_{B} \ldots n_{k} \ldots n_{N} \ldots n_{N}\right\rangle=n_{a}\left|n_{A} n_{B} \ldots n_{k} \ldots n_{N}\right\rangle$. Esta abordagem é a mesma utilizada em sistemas físicos para descrever cadeias de spin- 
$1 / 2$, e pode ser estendida para jogos com mais de jogadores e mais estratégias.

Afim de facilitar a identificação dos cenários, utilizamos uma regra de rótulo. A rotulação de cada perfil de estratégias é dada por meio da regra chamada "Littleendian" ${ }^{\text {, }}$ sendo definida pela regra, $|\mu\rangle=\left|n_{0} \cdot 2^{(0)}+n_{1} \cdot 2^{(1)}+\cdots+n_{N-1} \cdot 2^{(N-1)}\right\rangle$. Portanto, cada jogador é ordenado por um índice ${ }^{2} k$, o seu respectivo autovalor $n_{k}$ assume o papel de um "bit" na base binária. Para exemplificar esta regra, considere um cenário com 3 jogadores $(k \in\{0,1,2\})$; Se todos o jogadores cooperarem, todos os autovalores serão iguais à unidade, e portanto pela regra "Little-endian", temos o rótulo $\left|1 \cdot 2^{0}+1 \cdot 2^{1}+1 \cdot 2^{2}\right\rangle=|7\rangle$; Se somente o segundo jogador $(k=1)$ desertar $\left(n_{2}=0\right)$, teremos o rótulo $\left|1 \cdot 2^{0}+0 \cdot 2^{1}+1 \cdot 2^{2}\right\rangle=|5\rangle$.

\subsection{Modelo de spins para o Jogo do Bem Público}

Como descrito no segundo capítulo, o $J P B$ consiste de $N$ jogadores que adotam duas possíveis estratégias, cooperar ou desertar. Consideramos que este jogo pode ser representado por um sistema de $N$ spins com um grau de liberdade. A estratégia de cada jogador é então representada por uma variável discreta $n_{k}$, onde $k$ indexa cada jogador. Os possíveis valores de $n_{k}$ são ou 1 ou 0 , de modo que, 1 corresponde a estratégia cooperação, enquanto 0 corresponde a estratégia deserção. Além disso, número de jogadores é preservado entre as rodadas e, portanto, dizemos que cada jogador joga cada rodada com um número fixo de vizinhos. Os vizinhos de cada jogador são definidos por uma matriz adjacência. Esta matriz carrega a informação sobre a estrutura da rede de conexões entre os jogadores, podendo variar entre uma cadeia linear, onde cada jogador interage com somente dois outros jogadores, até uma rede completa, onde todos os jogadores interagem entre si a cada rodada. Todas demais estruturas de rede se dispõem entre os dois casos limites.

\footnotetext{
${ }^{1}$ A notação "Little-endian" é comumente utilizada na ciência da computação para rotular números binários na base decimal.

${ }^{2}$ Observe que o índice $k$ começa em 0 e termina em $N-1$, somando $N$ jogadores.
} 
Em sistemas físicos, definimos o operador Hamiltoniano $\hat{\mathcal{H}}$ para descrever a evolução temporal do sistema. Fazendo uma analogia ao operador Hamiltoniano, definimos o operador payoff individual $\hat{\varepsilon}_{k}$ para o JBP. Em seguida calculamos o operador ganho global $\hat{\varepsilon}$, resultado da soma de todos os operadores individuais. O operador $\hat{\varepsilon}_{k}$ é dado pela definição do Jogo do Bem Público (5.5). Fazemos aqui uma distinção entre o ganho de um jogador que coopera $\varepsilon_{k}^{C}$, e o ganho de um jogador que deserta $\varepsilon_{k}^{D}$. Esta distinção será conveniente quando analisarmos a influência do mecanismo de punição na próxima seção.

Antes de definir os principais operadores do $J B P$, relembramos as regras deste jogo. Dado $N$ jogadores, e duas estratégias, cada jogador pode cooperar pagando um custo $c>0$, ao bem público. O montante arrecado é acrescido por um juro $r$, tal que este juro esta sujeito a condição $(1<r<N-1)$. Como base nestas regras, definimos os operadores, ganho do cooperador, $\hat{\varepsilon}_{k}^{C}$, ganho do desertor, $\hat{\varepsilon}_{k}^{D}$, ganho individual, $\hat{\varepsilon}_{k}$ e ganho global, $\hat{\varepsilon}$,

$$
\begin{gathered}
\hat{\varepsilon}_{k}^{C}=c\left[\frac{r}{N} \sum_{j} \hat{n}_{j}-\hat{n}_{k}\right]\left(\hat{n}_{k}\right) . \\
\hat{\varepsilon}_{k}^{D}=c\left[\frac{r}{N} \sum_{j} \hat{n}_{j}-\hat{n}_{k}\right]\left(1-\hat{n}_{k}\right) . \\
\hat{\varepsilon}_{k}=\hat{\varepsilon}_{k}^{C}+\hat{\varepsilon}_{k}^{D} . \\
\hat{\varepsilon}_{k}=c\left[\frac{r}{N} \sum_{j} \hat{n}_{j}-\hat{n}_{k}\right] . \\
\hat{\varepsilon}=c(r-1) \sum_{k}^{N} n_{k} .
\end{gathered}
$$


Com os operadores ganho definidos, podemos analisar os resultados para todas as possíveis configurações de estratégias. Esta análise é feita com o operador ganho individual, e em seguida com o operador ganho global em um jogo com três jogadores $N=3$. Nesse jogo existem $2^{3}$ configurações de estratégias, com quatro distintos autovalores do operador ganho individual, e quatro distintos autovalores do operador ganho global. Os autovalores que correspondem aos ganhos dos cooperadores e dos desertores para cada perfil de estratégia são resumidos na tabela (5.8) para o operador ganho individual, e na tabela (5.9) para o operador ganho global,

\begin{tabular}{lcc}
\hline perfil & ganho do cooperador & ganho do desertor \\
\hline$|0\rangle$ & 0 & 0 \\
\hline$|1\rangle,|2\rangle,|4\rangle$ & $r c / 3-c$ & $r c / 3$ \\
\hline$|3\rangle,|5\rangle,|6\rangle$ & $2 r c / 3-c$ & $2 r c / 3$ \\
\hline$|7\rangle$ & $c(r-1)$ & 0 \\
\hline
\end{tabular}

Tabela 3: A tabela mostra os autovalores do operador ganho individual para os perfis de estratégias.

\begin{tabular}{lc}
\hline perfil & ganho de cada jogador \\
\hline$|0\rangle$ & 0 \\
\hline$|1\rangle,|2\rangle,|4\rangle$ & $c(r-1) / 3$ \\
\hline$|3\rangle,|5\rangle,|6\rangle$ & $2 c(r-1) / 3$ \\
\hline$|7\rangle$ & $c(r-1)$ \\
\hline
\end{tabular}

Tabela 4: A tabela mostra os autovalores do operador ganho global para as configurações de estratégias. 
Observamos que o maior autovalor do operador ganho individual e do operador ganho global é dado para perfis de estratégias diferentes, isto ocorre sempre, e é uma decorrência da condição imposta sobre os possíveis valores de $r$. Portanto, interpretamos esta divergência como o dilema do jogo. Como os jogadores adotam decisões egoístas, estes escolhem as suas estratégias baseando-se nos autovalores do operador ganho individual, o que leva ao pior cenário global. Pela a definição do Equilíbrio de Nash, a solução deste jogo é a deserção global. Devemos então analisar com cuidado, a escolha operador que melhor descreve o jogo.

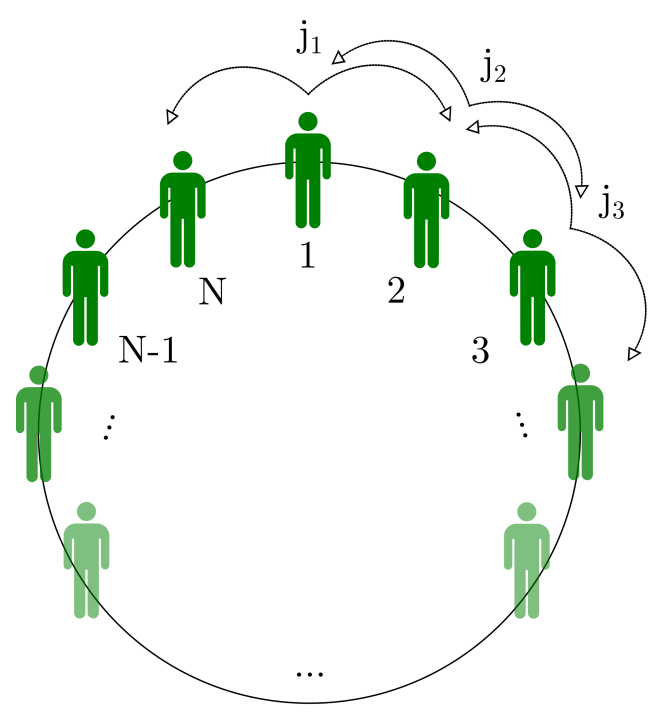

Figura 5.1: Arranjo dos jogadores em um linha completa fornece a condição de contorno periódica para o sistema ${ }^{4}$.

Variáveis globais são fundamentais para sistemas físicos, mas o mesmo não pode ser dito para a Teoria de Jogos, principalmente quando aplicamos o princípio de minimização. Inspirado na conexão entre os JPE e os sistemas Termodinâmicos, utilizamos o operador ganho global para descrever a dinâmica do sistema. Definimos o operador Hamiltoniano do sistema como $\hat{\mathcal{H}}=-\hat{\varepsilon}$. No contexto de um sistema físico, essa definição equivale a dizer que o sistema atinge o equilíbrio quando a energia total é minimizada. Fazemos, então, a simplificação $\Delta \equiv c(r-1)$ e calculamos a função de partição (5.10) e a estratégia média por jogador (5.11), a partir de (5.7) 
e (5.11) para uma rede circular de jogadores (5.1),

$$
Z=\operatorname{Tr}\left(e^{-\beta \hat{\mathcal{H}}}\right) \quad, \quad Z=\left(1+e^{\beta \Delta}\right)^{N}
$$

$$
\langle n\rangle=\frac{\partial}{\partial(\beta \Delta)} \ln (Z) \quad, \quad\langle n\rangle=\frac{1}{\left(1+e^{-\beta(\Delta)}\right)}
$$

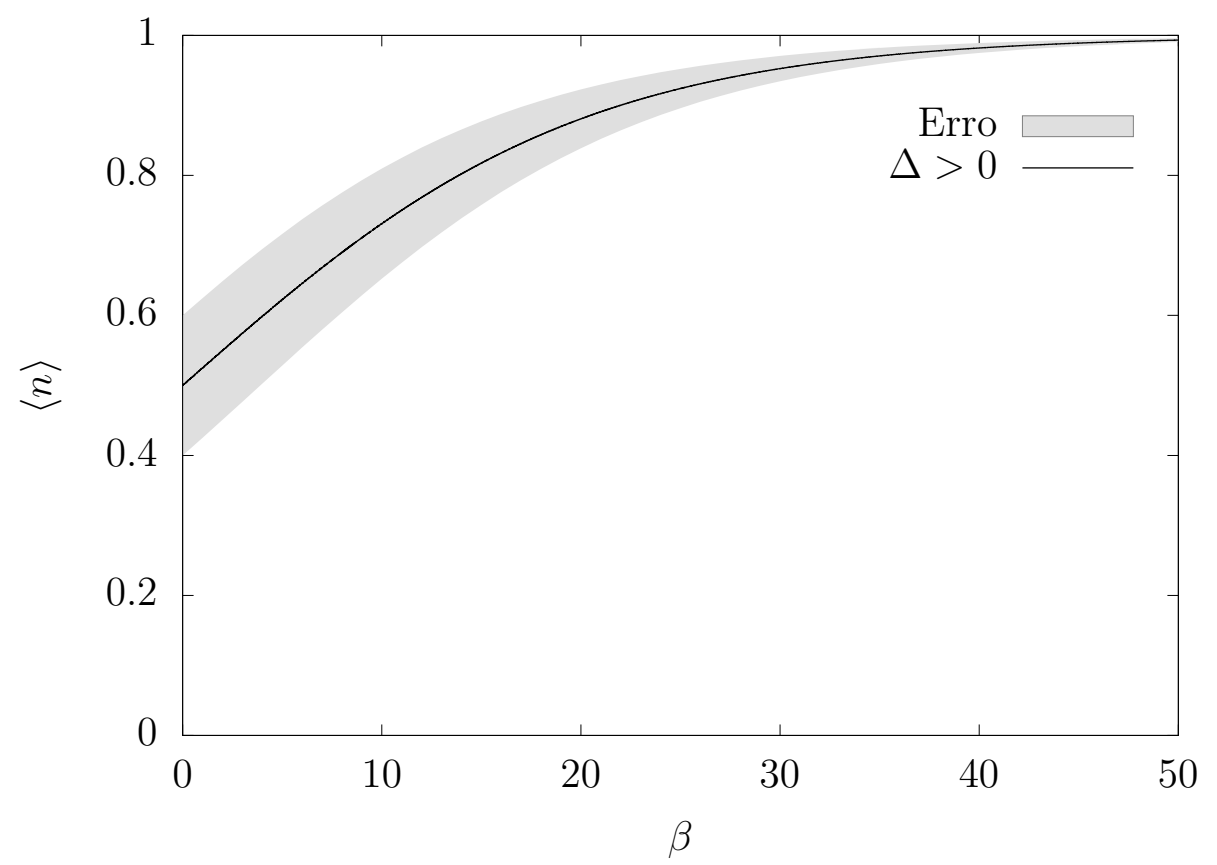

Figura 5.2: Observamos o crescimento monotônico da estratégia média por jogador, indicando que, para os valores $(\beta \gg 1)$ os jogadores tendem a cooperar. Este resultado não corrobora com o Equilíbrio de Nash previsto pela teoria para o JBP. Isto sugere que um fator intrínseco que afeta a decisão dos jogadores e corrige a dinâmica do jogo ${ }^{6}$. Em cinza é expresso a região de incerteza.

No gráfico (5.2) observamos que, para jogadores racionais $(\beta \rightarrow 1)$, vemos que a média das tende a cooperação, o que não corrobora com o EN previsto. O operador ganho global sugere que os jogadores priorizam somente o ganho, não considerando possíveis perdas. Assim como observamos na análise, perde-se a informação sobre o correto equilíbrio ao somar os operadores ganho individual. Esta inconsistência

\footnotetext{
${ }^{6}$ Imagem do artigo [44] $(C C B Y-N C 2.5)$.
} 
entre o resultado obtido e o $E N$. Esse resultado sugere que existe um mecanismo intrínseco que carrega a informação perdida sobre as possíveis perdas aos quais o jogadores estão sujeitos ao cooperar. Chamamos esta informação de risco de cooperação $\left(\mu_{k}\right)$, e que este contém o risco aos quais os jogadores estão sujeitos ao cooperar. O risco de cooperação está relacionado diretamente com Equilíbrio de Nash, pois pela definição do risco de cooperação sugere que no EN nenhum jogador consegue aumentar o seu ganho variando, de modo independente, a sua estratégia, dado que os seus adversários mantém as estratégias fixas. Portanto, definimos o risco de cooperação como (5.12).

$$
\mu_{k} \equiv-\frac{\partial\left\langle\varepsilon_{k}\right\rangle}{\partial\left\langle n_{k}\right\rangle}
$$

Considere o menor cenário do JBP com três jogadores, tal que o ganho individual esperado é dado por (5.13).

$$
\left\langle\hat{\varepsilon}_{k}\right\rangle=\frac{r c}{3}\left[\left\langle n_{k-1}\right\rangle+\left\langle n_{k}\right\rangle+\left\langle n_{k+1}\right\rangle\right]-c\left\langle n_{k}\right\rangle .
$$

Então, a correção feita pelo risco de cooperação neste cenário é dado por (5.14).

$$
\frac{\partial\left\langle\hat{\varepsilon}_{k}\right\rangle}{\partial\left\langle n_{k}\right\rangle}=\frac{r c}{3}-c \quad, \quad \mu_{k}=-\left[\frac{c r}{3}-c\right] .
$$

Para compreender melhor o comportamento do risco de cooperação, consideramos uma Hamiltoniana teste, dada exclusivamente pelo risco de cooperação. Essa Hamiltoniana teste é dada pelo operador linear $\hat{\mathcal{H}}_{\text {teste }}=\mu\left(\hat{n}_{k-1}+\hat{n}_{k}+\hat{n}_{k+1}\right)$. A análise dessa resulta em uma estratégia média $\langle n\rangle=\left[1+e^{-\beta \mu}\right]^{-1}$. Apesar desse resultado recuperar o $E N$ previsto pela teoria, esse sistema não descreve um cenário 
realístico, pois, a Hamiltoniana teste $\hat{\mathcal{H}}_{\text {teste }}$ favorece exclusivamente a minimização do risco, independente do valor do ganho. Escrevemos a Hamiltoniana que contém a informação completa do jogo por,

$$
\hat{\mathcal{H}}=-\sum_{k}^{N}\left(\hat{\varepsilon}_{k}-\mu_{k} \hat{n}_{k}\right) .
$$

Essa Hamiltoniana contém o incentivo do ganho e a informação sobre o Equilíbrio de Nash. Sob o ponto de vista termodinâmico, o risco de cooperação é análogo a um campo magnético externo, atuando sobre uma cadeia de spins.

$$
\hat{\mathcal{H}}=-(\Delta-\mu) \sum_{k}^{N} \hat{n}_{k}
$$

A adição do operador linear $\hat{n}_{k}$ com a constante de $\mu_{k}$ incorpora a condição EN. Portanto, a emergência da cooperação depende da relação entre o custo-ganho e o "risco de cooperação". Este é o resultado mais importante do nosso estudo. Temos, agora, a Hamiltoniana corrigida, $\hat{\mathcal{H}}_{k}$ considerando que o ganho é maximizado e o risco minimizado. Calculamos nova função de partição, e observamos agora que a densidade de estratégia adotada depende da diferença entre o ganho e o risco. Em um sistema aonde o risco $\mu$ é maior que o ganho $\Delta$ resgatamos o $E N$ correto, caso contrário observamos a emergência da cooperação. Esta análise é obtida ao analisarmos o gráfico da estratégia média adotada de acordo com os valroes de $\beta$ (5.3).

$$
Z=\left(1+e^{\beta(\Delta-\mu)}\right)^{N} \quad, \quad\langle n\rangle=\frac{1}{\left(1+e^{-\beta(\Delta-\mu)}\right)} .
$$




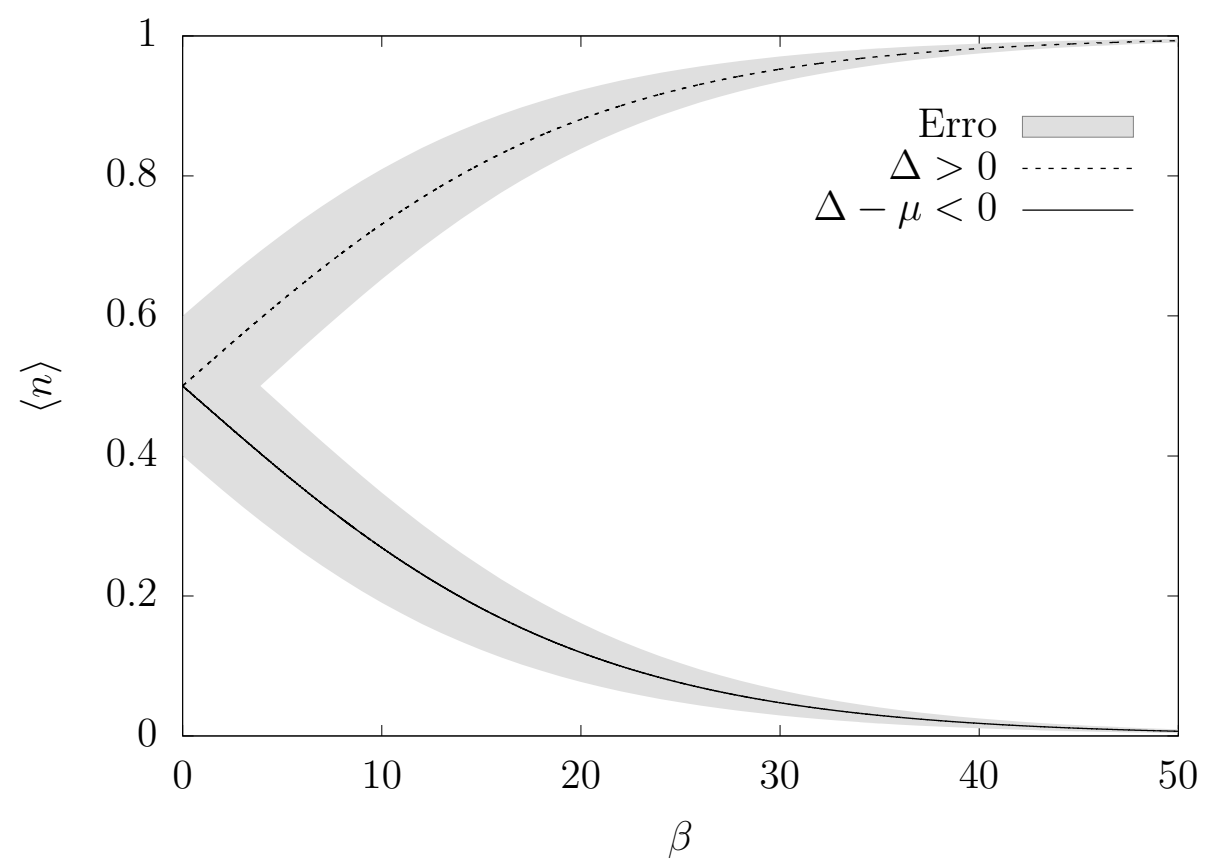

Figura 5.3: Estratégia média esperada quando o risco de cooperação é considerado ${ }^{8}$. Novamente observamos um comportamento monotonicamente, porém não exclusivamente crescente. A incerteza (SE) é representada pela região cinza.

\subsubsection{Jogo do Bem Público com punição aos desertores.}

A punição é um mecanismo de cooperação utilizado para desfavorecer desertores, portanto, um artifício assimétrico imposto a fim de promover a cooperação. Isso sugere que a punição modifica o comportamento dos jogadores para assegurar um número maior de cooperadores. Nesta seção examinamos o impacto da punição $(\gamma)$ sobre a dinâmica do $J B P$ em uma rede completa, visto na figura (5.4), utilizando a mesma técnica da seção anterior.

O operador ganho desertor com punição $\hat{\varepsilon}_{k}^{P}$, resulta da redução do autovalor $\hat{\varepsilon}_{k}^{D}$ do operador ganho desertor por um valor $\gamma$. O valor da punição pode variar entre 0 (sem punição) e 1 (punição máxima), $\gamma \in \Re / \gamma \in[0,1]$.

$$
\hat{\varepsilon}_{k}^{P}=(1-\gamma) \hat{\varepsilon}_{k}^{D}
$$

\footnotetext{
${ }^{8}$ Imagem retirada do artigo [44] $(C C B Y-N C 2.5)$.
} 


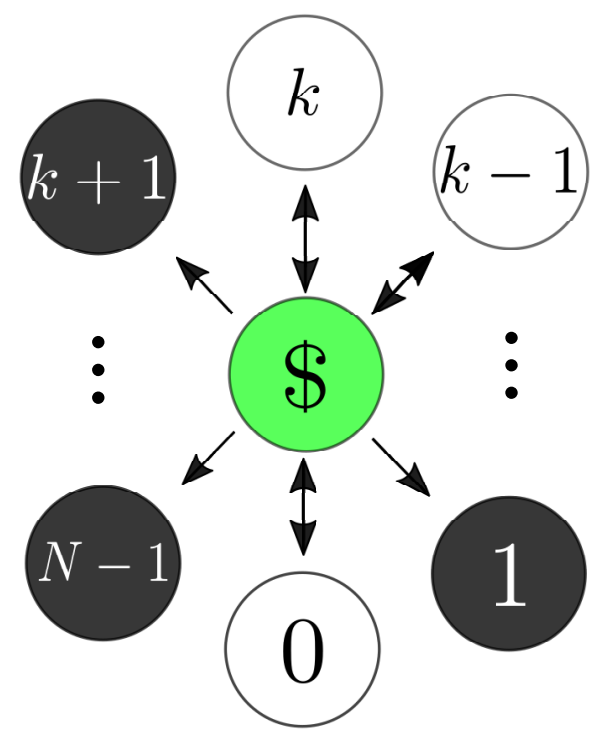

Figura 5.4: Representação esquemática do $J B P$ com punição para um grafo completo.

O novo operador ganho individual $\hat{\varepsilon}_{k}$ é calculado a partir do operador ganho cooperador com o novo operador ganho desertor com punição,

$$
\hat{\varepsilon}_{k}=\hat{\varepsilon}_{k}^{C}+\hat{\varepsilon}_{k}^{P}
$$

Definimos $(b \equiv r c)$ e sabendo que o operador número é nilpotente $\left(\hat{n}_{k}\right)^{2} \equiv \hat{n}_{k}$,

$$
\hat{\varepsilon}_{k}=\frac{b}{N} \sum_{\ell=0}^{N-1} \hat{n}_{\ell}\left(1-\gamma\left(1-\hat{n}_{k}\right)\right)-c \hat{n}_{k}
$$

Sabemos que o EN precisa ser satisfeito. Baseado nos resultados do caso sem punição, introduzimos o risco de cooperação,

$$
\begin{gathered}
\mu_{k}^{p}=\frac{\partial\left\langle\varepsilon_{k}\right\rangle}{\partial\left\langle n_{k}\right\rangle}=\frac{\partial}{\partial\left\langle n_{k}\right\rangle}\left[\frac{b}{N} \sum_{\ell=0}^{N-1}\left\langle n_{\ell}\right\rangle\left(1-\gamma\left(1-\left\langle n_{k}\right\rangle\right)\right)-c\left\langle n_{k}\right\rangle\right] \\
\mu_{k}^{p}=\mu_{k}-\frac{b \gamma}{N} \sum_{j \neq k}^{N-1}\left\langle n_{\ell}\right\rangle
\end{gathered}
$$


Calculamos o operador Hamiltoniano com punição $\hat{\mathcal{H}}^{P}$,

$$
\begin{gathered}
\hat{\mathcal{H}}^{P}=-\left(\hat{\varepsilon}_{k}-\mu_{k}^{P} \hat{n}_{k}\right) \\
\left.\hat{\mathcal{H}}^{P}=-\frac{\gamma b}{N} \sum_{\ell, k=0}^{N-1} \hat{n}_{\ell} \hat{n}_{k}-\sum_{k=0}^{N-1}\left(\frac{b}{N}\right) \sum_{\ell=0}^{N-1} \hat{n}_{\ell}-c \hat{n}_{k}-b \gamma-\mu_{k}^{p}\right) \hat{n}_{k},
\end{gathered}
$$

Simplificamos os termos, $\hat{N}^{2}=\sum_{\langle\ell, k\rangle}^{N-1} \hat{n}_{\ell} \hat{n}_{k}$, e, $\hat{N}=\sum_{k=0}^{N-1} \hat{n}_{k}$ (5.26), e definimos as constantes de acoplamento e "campo magnético", $\alpha_{2}=\frac{\gamma b}{N}$ e $\alpha_{1}=\left(h_{k}^{p}-\mu_{k}^{p}\right)$ respectivamente. As constantes de acoplamento no termo de dois corpos $\sum_{\langle k, \ell\rangle}^{N} \hat{n}_{k} \hat{n}_{\ell}$ são iguais. Esta característica permite que apliquemos a aproximação de campomédio. Estimamos uma variação no ganho $(\delta \varepsilon)$ devido a punição por meio da aproximação de campo médio, $\delta \varepsilon \approx-\gamma b\langle n\rangle[1-\langle n\rangle]$. Portanto, ao mesmo tempo que a punição favorece o comportamento cooperativo reduzindo o ganho do desertor em $\gamma$, esta também favorece a deserção para cenários em que a relação custo-ganho é muito baixa. Este resultado recupera os resultados relatados na literatura $[59,77,78]$ para jogos iterados.

Investigamos o risco de cooperação com punição $\mu_{k}^{p}$ substituindo a média local $\left\langle n_{k}\right\rangle$ pela média global $\langle n\rangle$,

$$
\mu_{k}^{p} \approx \mu_{k}-\gamma b\langle n\rangle(N-1) / N
$$

Substituímos $\langle n\rangle$ na equação (5.25) por $\bar{n}$ dando pela equação (5.17),

$$
\mu^{p}=\mu-\gamma b\langle n\rangle(N-1) \quad, \quad \hat{\mathcal{H}}^{P}=-\alpha_{2} \hat{N}^{2}-\alpha_{1} \hat{N}
$$

Escrevemos o operador Hamiltoniano como (5.26) $\operatorname{com} \hat{N}^{2}=\sum_{k=\langle(k, \ell)\rangle} \hat{n}_{k} \hat{n}_{\ell}$,

\footnotetext{
${ }^{10}$ Imagem retirada do artigo [44] $(C C B Y-N C 2.5)$.
} 


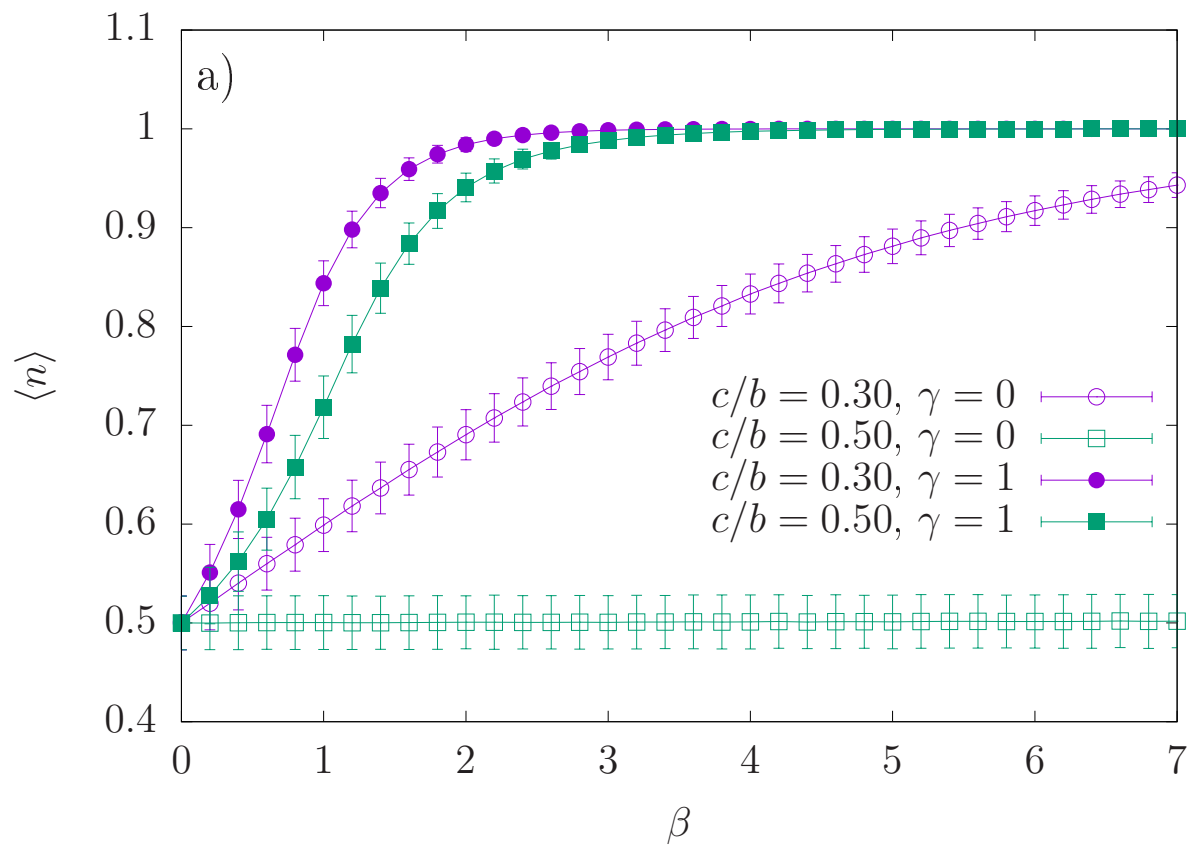

Figura 5.5: O gráfico mostra o comportamento da estratégia média por jogador com a redução do ruído. Percebe-se que a punição promove a cooperação, inclusive no cenário de indecisão $c / b=0.5^{10}$.

$\hat{N}=\sum_{k} \hat{n}_{k}$ e acoplamentos $\alpha_{2}=\gamma b / N$ e $\alpha_{1}=(\Delta-c+b / N)-\gamma b(1-\bar{n} / N)$. A função de partição correspondente é calculada,

$$
Z(x)=z_{0}(x) \sum_{k=0}^{\infty}\left[\frac{\left(\beta \alpha_{2}\right)^{k}}{k !} \frac{1}{Z_{0}(x)} \frac{\partial^{2 k}}{\partial(x)^{2 k}} Z_{0}(x)\right]
$$

Tal que $x=\beta \alpha_{1}$ e $Z_{0}(x)=\left(1+e^{x}\right)^{N}$. Uma formulação mais simples do operador $(\partial / \partial x)^{2 k}$ é obtida por meio da mudança de variável $(\partial / \partial x)^{2 k}=\sum_{\ell=0}^{2 k}\left\{\begin{array}{c}2 k \\ \ell\end{array}\right\}(u-$ $1)^{\ell}(\partial / \partial)^{\ell}$. O simbolo $\left\{\begin{array}{c}2 k \\ \ell\end{array}\right\}$ denota os números de Stirling de segundo tipo para $\ell \leq 2 k$, e 0 caso contrário.

Nas figuras (5.5) e (5.6), mostramos que os efeitos da punição no JBP em simulações de Monte Carlo com o algoritmo de Metropolis-Hastings com $N=1024$ jogadores em um grafo completo. Para $b>2 c$ e valores positivos de $\gamma$, a punição favorece a cooperação. No caso especial $b=2 c$ revela a equivalência entre cooperação e deserção cooperativas, sem punição. A adição da punição na dinâmica do jogo

\footnotetext{
${ }^{12}$ Imagem retirada do artigo [44] (CCBY-NC 2.5) .
} 


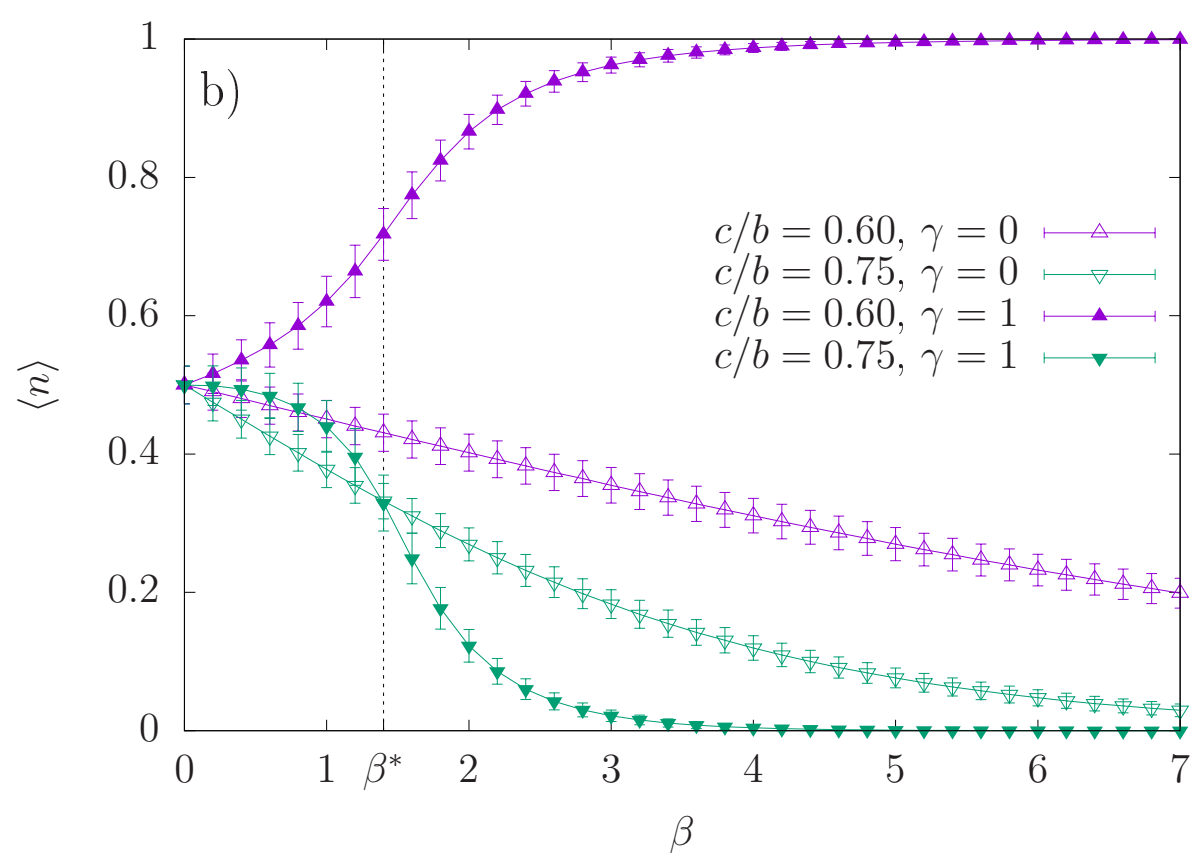

Figura 5.6: O gráfico mostra o comportamento da estratégia média por jogador para os valores $c / b \sim 0.65$. O gráfico mostra que a punição muda abruptamente o comportamento dos jogadores. Para valores $c / b>0.75$ e $\beta>\beta^{*} \sim 1.3$ a punição passa a promover a deserção ${ }^{12}$.

favorece estratégias cooperativas, isto pode ser observado na figura (5.6). Realizamos simulações de Monte Carlo usando o algoritmo Metropolis [73, 79].

A figura (5.7) descreve o comportamento de $\langle n\rangle$ para $c_{0}=0.664 b$ e $c_{1}=$ 0.665b, com $N=2^{10}$ jogadores e parâmetro de punição $\gamma=1$. No primeiro caso a punição favorece a cooperação e a densidade de cooperadores $(\langle n\rangle)$ aumenta monotônica e continuamente para valores de $\beta \rightarrow \infty$. No segundo caso, fazemos um pequeno incremento sobre o custo anterior, $c_{1}=c_{0}+\delta c$ com $\delta c=10^{-3}$. Esta pequena variação no custo altera o comportamento de $\langle n\rangle$ para $\beta>2.08$. Os resultados numéricos na figura (5.7) sugerem que $\langle n\rangle$ desenvolve uma descontinuidade $\Gamma_{c}=1$, em torno de $c \approx 2 / 3$ e $\gamma=1$, com a deserção sendo a estratégia mais vantajosa para os jogadores racionais. A analogia com a termodinâmica sugere a interpretação de $c$ e $\langle n\rangle$ como o campo magnético $B$ e a densidade de magnetização

\footnotetext{
${ }^{14}$ Figura retirada do artigo [44] (CC BY-NC 2.5).

${ }^{16}$ Figura retirada do artigo [44] ( $\left.C C B Y-N C 2.5\right)$.

${ }^{18}$ Imagem retirada do artigo [44] (CCBY-NC 2.5) .
} 


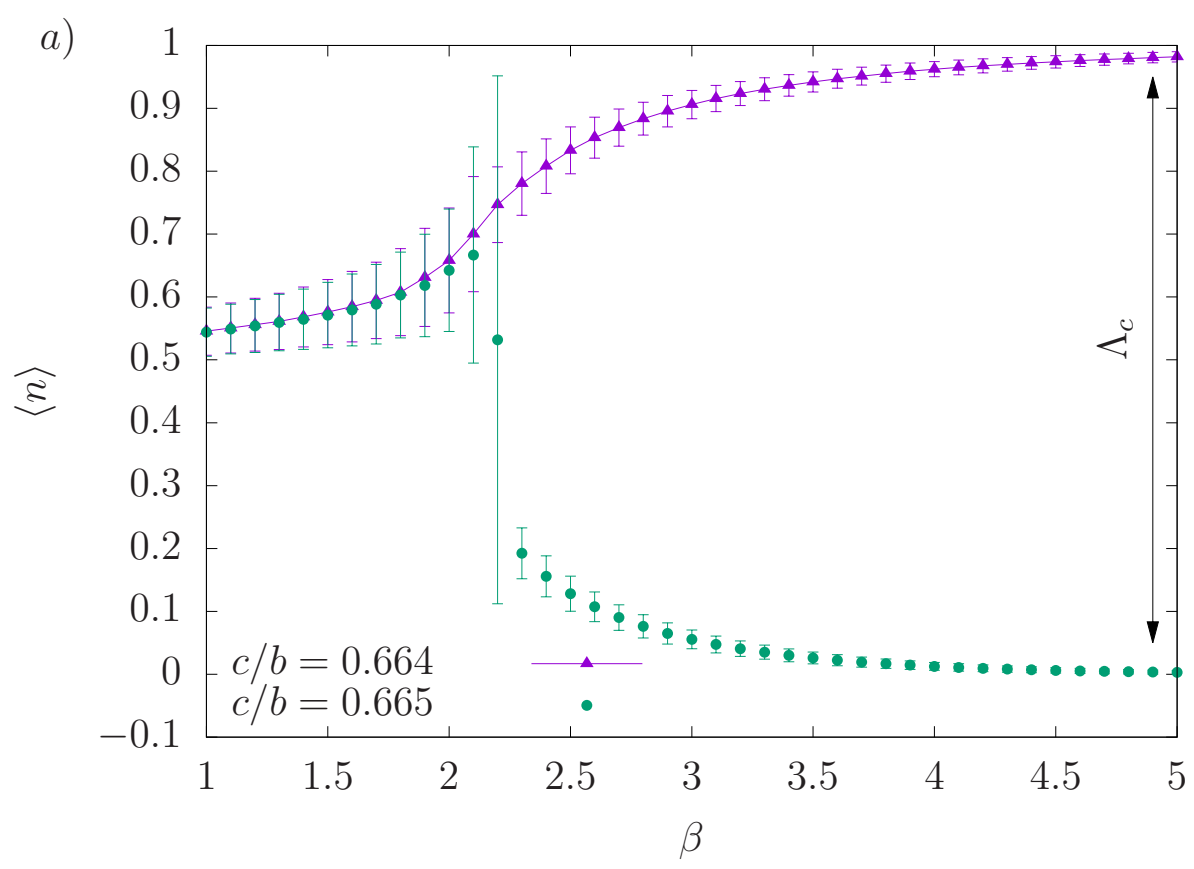

Figura 5.7: A análise do espaço de parâmetros custo-ganho com punição máxima $\gamma=1$ no limiar de um mudança de regimes cooperação-deserção, revela um sinal de uma possível transição de fase crítica. A condição de transição de fase crítica é observada após uma pequena redução na relação custo-ganho com punção máxima $\gamma=1^{14}$.

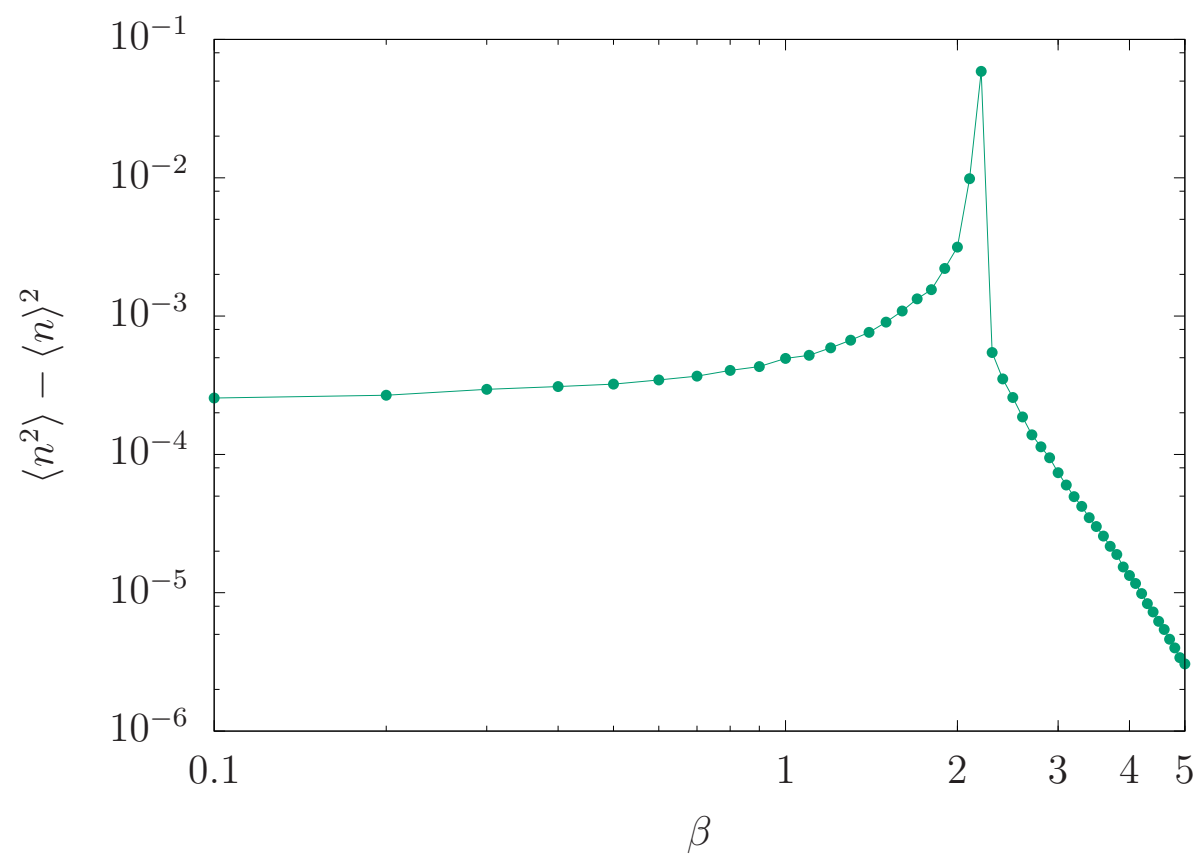

Figura 5.8: O gráfico mostra o comportamento da variância da densidade de estratégias com o aumento de $\beta$ na escala logarítmica. Observamos o surgimento da transição de fase para a condição vista anteriormente $(c / b=0.665)$ com punição máxima $(\gamma=1)$ com o aumento de $\beta$. A variância revela que a divergência ocorre para valores próximos de $\beta \sim 2^{16}$. 


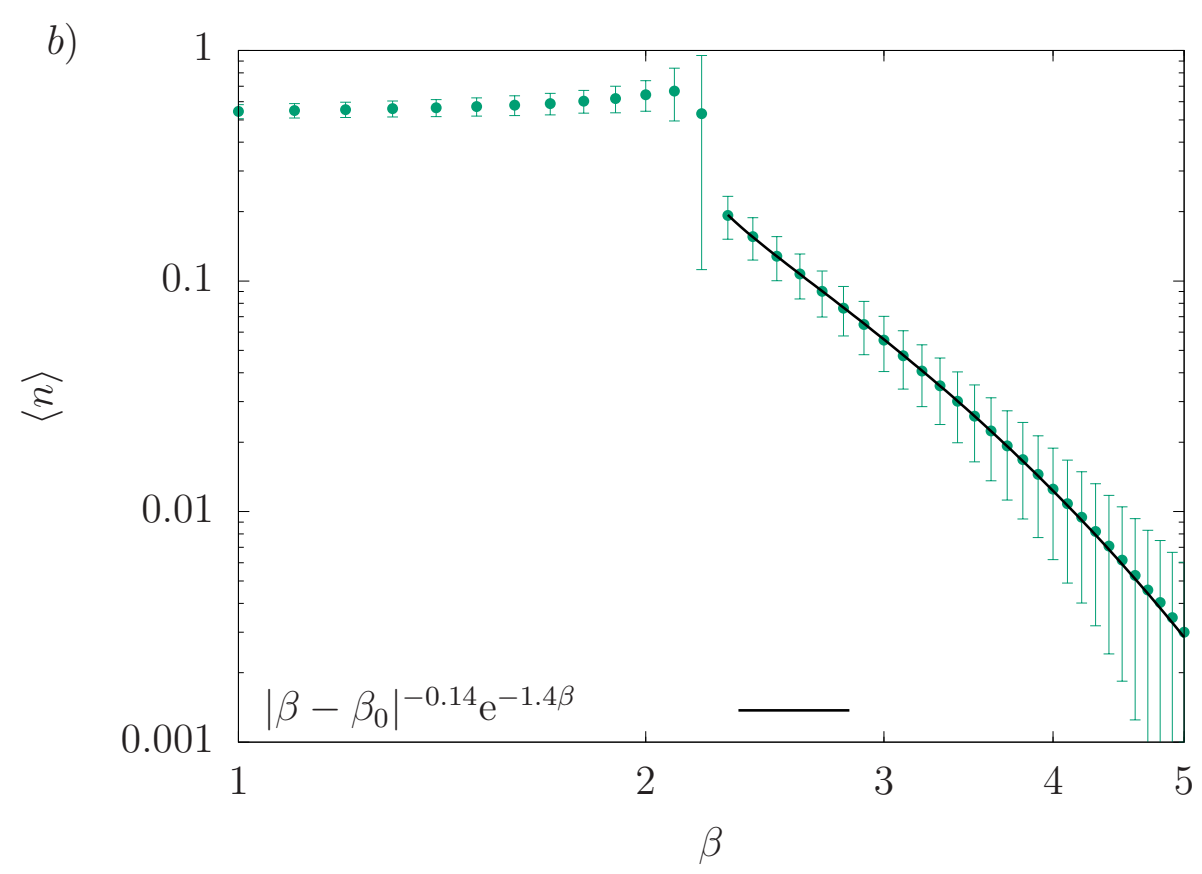

Figura 5.9: Comportamento da variância na escala log-log. O gráfico mostra uma transição de fase tipo lambda que decai com a lei $\left|\beta-\beta_{0}\right|^{-\omega_{1}} e^{-\omega_{2} \beta 18}$.

$m$, respectivamente, de modo que $\left.(\partial m / \partial B)\right|_{\beta, \gamma}=\Lambda_{c}$. Este comportamento sugere uma transição de fase de primeira ordem, separando o regime cooperativo $\left(c<c_{1}\right)$ do regime não cooperativo $\left(c \geq c_{1}\right)$ para jogadores racionais. Além disso, ressaltamos que $\langle n\rangle$ muda rapidamente com $\beta$. Uma análise na escala semilog e log-log mostra que a densidade de cooperadores $\langle n\rangle$ decai com a lei $\left|\beta-\beta_{0}\right|^{-\omega_{1}} e^{-\omega_{2} \beta}$, com $\beta_{0}=2.175, \omega_{1}=0.140 \pm 0.002$ e $\beta_{2}=1.400 \pm 0.005 \approx 10 \omega_{1}$. A flutuação $\left\langle n^{2}\right\rangle-\langle n\rangle^{2}$ exibe uma transição do tipo lambda, $\lambda$, em escala log-log, como mostra a figura (5.8), com um pico em torno de $\beta \approx 2.2$. Os detalhes da transição observada e, se os pontos críticos são únicos ou múltiplos (ocorrendo ao longo de uma linha crítica) não é inteiramente claro neste momento. 


\section{6}

\section{Conclusão}

Todos concordamos que a sua teoria é louca, mas é louca o suficiente?"

- Niels Bohr

Neste trabalho apresentamos as ferramentas analíticas da Mecânica Estatística e da Termodinâmica que são utilizadas para descrever a dinâmica dos jogos potenciais evolucionários na teoria dos jogos. Apresentamos, também, uma formulação quantitativa para o Jogo do Bem Público, mostrando que a introdução do risco de cooperação é fundamental para a correta descrição do jogo. O risco de cooperação é analago a um operador de um corpo e introduz a informação sobre o Equilíbrio de Nash, ou seja, sobre o risco que os jogadores estão sujeitos ao cooperar. O nossos resultados numéricos mostram que o $J B P$ apresenta uma transição de fase de primeira ordem, separando a fase cooperativa da não cooperativa. Outra transição de fase é sugerida ao longo do parâmetro de racionalidade, $\beta$, para um valor específico de $c / b$ e $\gamma$. A classificação e o estudo detalhado sobre a transição de fase e o parâmetro gama está em estudo. Notamos que jogos com estruturas espaciais mais complexas recriam as condições para o comportamento cooperativo competitivo da literatura. Consideramos este estudo como um avanço na área dos jogos potenciais, lembrando que consideramos apenas as estruturas mais simples para o $J B P$, com 
todos os jogadores interagindo entre si. O objetivo dessa estrutura é, em primeiro lugar, fornecer um modo direto de comparação dos resultados obtidos aqui com os resultados já conhecidos na literatura, e em segundo lugar, a emergência da cooperação devido a estruturas espaciais deturpar a análise sobre "risco de cooperação". Os efeitos exatos do risco de cooperação com estruturas espaciais complexas ainda estão sob investigação e merecem mais estudos. 


\section{Referências Bibliográficas}

[1] A. Świerniak, M. Krześlak, S. Student, and J. Rzeszowska-Wolny, "Development of a population of cancer cells: Observation and modeling by a Mixed Spatial Evolutionary Games approach," Journal of Theoretical Biology, 2016. 1, 10, 15

[2] I. P. Tomlinson, "Game-theory models of interactions between tumour cells," European Journal of Cancer Part A, 1997.

[3] L. Dugatkin and H. Reeve, Game Theory $\&$ Animal Behavior. Oxford University Press, 1998.

[4] D.-J. F.G., C. M., I. S.N., L. D., and S.-L. P.C., "The structure of a bottlenose dolphin society is coupled to a unique foraging cooperation with artisanal fishermen," Biology Letters, 2012.

[5] C. Packer and A. E. Pusey, "Cooperation and competition within coalitions of male lions: kin selection or game theory?," Nature, 1982. 5, 14

[6] J. M. Pacheco, F. C. Santos, and D. Dingli, "The ecology of cancer from an evolutionary game theory perspective," Interface Focus, 2014.

[7] E. Pennisi, "On the Origin of Cooperation," Science, 2009. 1, 10, 15

[8] A. Wallace, Contributions to the Theory of Natural Selection: A Series of Essays - Scholar's Choice Edition. Creative Media Partners, LLC, 2015. 1, 2

[9] R. Pool, "Putting game theory to the test," Science, 1995. 2, 14 
[10] S. E. Riechert, "Games spiders play: Behavioral variability in territorial disputes," Behavioral Ecology and Sociobiology, 1978. 4, 5

[11] S. Riechert, "Spiders as Biological Control Agents," Annual Review of Entomology, 1984. 2, 5, 14

[12] C. Darwin and R. Stauffer, Charles Darwin's Natural Selection: Being the Second Part of His Big Species Book Written from 1856 to 1858. Cambridge University Press, 1987. 2

[13] J. V. Neumann and O. Morgenstern, "Theory of Games and Economic Behaviour," The Mathematical Gazette, 2007. 2, 3, 10, 12, 13, 14

[14] J. M. Smith and G. R. Price, "The logic of animal conflict," Nature, 1973. 3, 20

[15] H. Gintis, Game Theory Evolving. 2019. 3, 9, 15

[16] H. R. Varian, R. J. Aumann, and S. Hart, "Handbook of Game Theory: With Economic Applications, Volume I.," The Economic Journal, 2006. 3, 4, 5

[17] J. F. Nash, "The Bargaining Problem," Econometrica, 2006. 3

[18] G. Szabó and G. Fáth, "Evolutionary games on graphs," 2007. 5, 10, 19, 28, $29,33,34$

[19] G. Hardin, "The tragedy of the commons," in Environmental Ethics for Engineers, 2018. 3, 16, 17

[20] J. Nash, "Two-Person Cooperative Games," Econometrica, 2006. 4, 15

[21] J. Nash, "Non-Cooperative Games," Annals of Mathematics, vol. 75, no. 3, pp. $452-466,1951.15$ 
[22] J. F. Nash, "Games, Equilibrium Points in n-Person," Proceedings of the National Academy of Sciences of the United States of America, vol. 36, no. 1, pp. $48-49,1950.4$

[23] J. Maynard Smith and P. G.R., "The Logic of Animal Conflict," Nature, vol. 246, no. 1l, pp. 150-018, 1973. 4, 19

[24] J. M. Smith, Evolution and the theory of games, vol. 31. 1983. 4

[25] C. L. Apicella, F. W. Marlowe, J. H. Fowler, and N. A. Christakis, "Social networks and cooperation in hunter-gatherers.," Nature, 2012. 4

[26] G. Szabó and I. Borsos, "Evolutionary potential games on lattices," 2016. 5, $10,19,28,29,33,34$

[27] R. Selten, "Reexamination of the perfectness concept for equilibrium points in extensive games," International Journal of Game Theory, 1975. 5

[28] H. A. Simon, "A Behavioral Model of Rational Choice," The Quarterly Journal of Economics, 1955. 5

[29] M. Perc, J. J. Jordan, D. G. Rand, Z. Wang, S. Boccaletti, and A. Szolnoki, "Statistical physics of human cooperation," 2017. 5, 19

[30] C. Castellano, S. Fortunato, and V. Loreto, "Statistical physics of social dynamics," Reviews of Modern Physics, 2009.

[31] C. Adami and A. Hintze, "Thermodynamics of evolutionary games," Physical Review E, 2018. 19, 29, 33

[32] M. A. Amaral and M. A. Javarone, "Heterogeneous update mechanisms in evolutionary games: Mixing innovative and imitative dynamics," Physical Review E, 2018. 
[33] M. A. Amaral, M. Perc, L. Wardil, A. Szolnoki, E. J. Da Silva Júnior, and J. K. Da Silva, "Role-separating ordering in social dilemmas controlled by topological frustration," Physical Review E, 2017.

[34] A. Szolnoki, G. Szabó, and M. Perc, "Phase diagrams for the spatial public goods game with pool punishment," Physical Review E - Statistical, Nonlinear, and Soft Matter Physics, 2011. 19

[35] L. Wardil and M. A. Amaral, "Cooperation in public goods games: Stay, but not for too long," Games, vol. 8, no. 3, 2017. 5

[36] R. Pool, "Economics: Game Theory's Winning Hands," Science, vol. 266, no. 5184 , p. $371,1994.5$

[37] M. A. Pereira and A. S. Martinez, "Pavlovian Prisoner's Dilemma-Analytical results, the quasi-regular phase and spatio-temporal patterns," Journal of Theoretical Biology, 2010. 5

[38] M. A. Pereira, "Exhaustive exploration of Prisoner's Dilemma Parameter space in one-dimensional cellular automata," Brazilian Journal of Physics, 2008. 23

[39] F. M. Oliveira, M. A. Pereira, and A. S. Martinez, "O dilema do prisioneiro nas redes uni, bi e tridimensional," in Simpósio Internacional de Iniciação Científica (SIICUSP), USP, 2010.

[40] F. M. d. Oliveira and A. S. Martinez, "Dilema do prisioneiro nas redes unidimensional e bidimensional," in Simpósio Internacional de Iniciação Científica (SIICUSP), USP, 2008.

[41] M. A. Pereira, A. L. Espíndola, and A. S. Martinez, "Dilema do prisioneiro no autômato celular unidimensional: exploração exaustiva do espaço de parâmetros 
para estratégia darwiniana e pavloviana," in Encontro Nacional de Física da Matéria Condensada (ENFMC), SBF, 2008.

[42] R. Oliveira dos Santos Soares and A. S. Martinez, "The geometrical patterns of cooperation evolution in the spatial prisoner's dilemma: An intra-group model," Physica A: Statistical Mechanics and its Applications, 2006. 5

[43] L. E. Blume, "The Statistical Mechanics of Strategic Interaction," Games and Economic Behavior, 1993. 6, 19, 30

[44] G. M. Nakamura, G. S. Contesini, and A. S. Martinez, "Cooperation risk and Nash equilibrium: Quantitative description for realistic players," Physica A: Statistical Mechanics and its Applications, vol. 515, pp. 102-111, 2019. 6, 19, $28,29,34,41,44,46,47,48$

[45] R. Gibbons, Game Theory for Applied Economists. 2019. 10, 11, 15

[46] M. Cantor, P. C. Simões-Lopes, and F. G. Daura-Jorge, "Spatial consequences for dolphins specialized in foraging with fishermen," Animal Behaviour, 2018. 10

[47] C. M. Russell and H. A. Simon, "Administrative Behavior," The American Journal of Nursing, 2006. 12

[48] J. Sensat, "Game theory and rational decision," Erkenntnis, 1997. 12

[49] J. Bentham and J. Bentham, "An Introduction to the Principles of Morals and Legislation," in The Collected Works of Jeremy Bentham: An Introduction to the Principles of Morals and Legislation, 2015. 12

[50] C. Hauert and G. Szabó, "Game theory and physics," American Journal of Physics, 2005. 16 
[51] C. Hauert, S. De Monte, J. Hofbauer, and K. Sigmund, "Replicator dynamics for optional public good games," Journal of Theoretical Biology, 2002.

[52] H. Brandt, C. Hauert, and K. Sigmund, "Punishment and reputation in spatial public goods games," Proceedings of the Royal Society B: Biological Sciences, 2003.

[53] G. Szabó and C. Hauert, "Phase Transitions and Volunteering in Spatial Public Goods Games," Physical Review Letters, 2002. 16

[54] S. D. Levitt and J. A. List, "What Do Laboratory Experiments Measuring Social Preferences Reveal About the Real World?," Journal of Economic Perspectives, 2007. 17

[55] I. Pavlov and G. Anrep, Conditioned Reflexes. Dover Publications, 2012. 19

[56] M. Nowak, Evolutionary Dynamics. Harvard University Press, 2006. 19, 23

[57] J. Hofbauer, K. Sigmund, and P. Sigmund, Evolutionary Games and Population Dynamics. Cambridge University Press, 1998. 20, 21, 23, 29

[58] K. Sigmund, "William D. Hamilton's Work in Evolutionary Game Theory," Theoretical Population Biology, vol. 59, no. 1, pp. 3-6, 2001. 20

[59] R. M. May, "The evolution of cooperation," Nature, 1981. 19, 29, 46

[60] M. Perc, "Chaos promotes cooperation in the spatial prisoner's dilemma game," Europhysics Letters, 2006. 19, 28, 29, 33, 34

[61] G. Szabó and C. Tőke, “Evolutionary prisoner's dilemma game on a square lattice," Physical Review E - Statistical Physics, Plasmas, Fluids, and Related Interdisciplinary Topics, 1998. 28, 29, 33, 34 
[62] A. Hintze and C. Adami, "Punishment in public goods games leads to metastable phase transitions and hysteresis," Physical Biology, 2015.

[63] A. McAvoy, N. Fraiman, C. Hauert, J. Wakeley, and M. A. Nowak, "Public goods games in populations with fluctuating size," Theoretical Population Biology, 2018.

[64] L. E. Blume, "How noise matters," Games and Economic Behavior, 2003. 19, 30

[65] J. Maynard Smith, "The theory of games and the evolution of animal conflicts," Journal of Theoretical Biology, 1974. 20

[66] R. M. May, "Simple mathematical models with very complicated dynamics," in Universality in Chaos, Second Edition, 2017. 21

[67] M. A. Nowak and R. M. May, "Evolutionary games and spatial chaos," Nature, 1992. $21,25,29$

[68] R. Axelrod, The Evolution of Cooperation: Revised Edition. Basic Books, 2009. 25

[69] L. A. Imhof, D. Fudenberg, and M. A. Nowak, "Tit-for-tat or win-stay, loseshift?," Journal of Theoretical Biology, 2007. 29

[70] M. A. Nowak and K. Sigmund, "The alternating prisoner's dilemma," Journal of Theoretical Biology, 1994. 29

[71] L. Reichl, A Modern Course in Statistical Physics. Physics textbook, Wiley, 2009. 30

[72] R. J. Glauber, "Time-dependent statistics of the Ising model," Journal of Mathematical Physics, 1963. 30, 32 
[73] D. P. Landau and K. Binder, A Guide to Monte Carlo Simulations in Statistical Physics. 2014. 32, 48

[74] M. E. J. Newman and G. T. Barkema, Monte Carlo Methods in Statistical Physics. 1999.

[75] W. K. Hastings, "Monte carlo sampling methods using Markov chains and their applications," Biometrika, 1970. 30, 32

[76] R. Baxter, Exactly Solved Models in Statistical Mechanics. Elsevier Science, 2016. 31

[77] K. Sigmund, C. Hauert, and M. A. Nowak, "Reward and punishment," Proceedings of the National Academy of Sciences, vol. 98, no. 19, pp. 10757-10762, 2001. 46

[78] D. G. Rand, A. Dreber, T. Ellingsen, D. Fudenberg, and M. A. Nowak, "Positive interactions promote public cooperation," Science, 2009. 46

[79] H. J. Rothe, Lattice Gauge Theories: An Introduction. 2005. 48 